\title{
Platelet-derived miR-223 promotes a phenotypic switch in arterial injury repair
}

\author{
Zhi Zeng', Luoxing Xia, ${ }^{1}$ Xuejiao Fan, ${ }^{1}$ Allison C. Ostriker, ${ }^{2}$ Timur Yarovinsky, ${ }^{2}$ Meiling Su, ${ }^{1}$ Yuan Zhang, ${ }^{1}$ Xiangwen Peng, ${ }^{1}$ Yi Xie, ${ }^{2}$ \\ Lei Pi, ${ }^{3}$ Xiaoqiong Gu, ${ }^{4}$ Sookja Kim Chung, ${ }^{5}$ Kathleen A. Martin, ${ }^{2}$ Renjing Liu, ${ }^{6,7}$ John Hwa, ${ }^{2}$ and Wai Ho Tang ${ }^{1}$ \\ Institute of Pediatrics, Guangzhou Women and Children's Medical Center, Guangzhou Medical University, Guangzhou, China. Yale Cardiovascular Research Center, Section of Cardiovascular Medicine, \\ Department of Internal Medicine, Yale School of Medicine, New Haven, Connecticut, USA. '3epartment of Clinical Biological Resource Bank, Institute of Pediatrics, Guangzhou Women and Children's Medical \\ Center, Guangzhou Medical University, Guangzhou, China. `Department of Clinical Biological Resource Bank, Department of Clinical Laboratory, Institute of Pediatrics, Guangzhou Women and Children's \\ Medical Center, Guangzhou Medical University, Guangzhou, China. ${ }^{5}$ School of Biomedical Sciences, University of Hong Kong, Hong Kong, China. ${ }^{6}$ Agnes Cinges Laboratory for Diseases of the Aorta, Centenary \\ Institute, and `Sydney Medical School, University of Sydney, Sydney, New South Wales, Australia.
}

\begin{abstract}
Upon arterial injury, endothelial denudation leads to platelet activation and delivery of multiple agents (e.g., TXA2, PDCF), promoting VSMC dedifferentiation and proliferation (intimal hyperplasia) during injury repair. The process of resolution of vessel injury repair, and prevention of excessive repair (switching VSMCs back to a differentiated quiescent state), is poorly understood. We now report that internalization of APs by VSMCs promotes resolution of arterial injury by switching on VSMC quiescence. Ex vivo and in vivo studies using lineage tracing reporter mice (PF4-cre $\times \mathrm{mT} / \mathrm{mG}$ ) demonstrated uptake of GFPlabeled platelets $(\mathrm{mG})$ by mTomato red-labeled VSMCs $(\mathrm{mT})$ upon arterial wire injury. Genome-wide miRNA sequencing of VSMCs cocultured with APs identified significant increases in platelet-derived miR-223. miR-223 appears to directly target PDGFRß (in VSMCs), reversing the injury-induced dedifferentiation. Upon arterial injury, platelet miR-223-KO mice exhibited increased intimal hyperplasia, whereas miR-223 mimics reduced intimal hyperplasia. Diabetic mice with reduced expression of miR-223 exhibited enhanced VSMC dedifferentiation and proliferation and increased intimal hyperplasia. Our results suggest that horizontal transfer of platelet-derived miRNAs into VSMCs provides a novel mechanism for regulating VSMC phenotypic switching. Platelets thus play a dual role in vascular injury repair, initiating an immediate repair process and, concurrently, a delayed process to prevent excessive repair.
\end{abstract}

\section{Introduction}

At sites of vascular injury where endothelium is damaged or removed, vascular smooth muscle cells (VSMCs) are directly exposed to activated platelets (APs) $(1,2)$. Platelets provide a first and crucial line of defense against vascular injury to maintain normal hemostasis $(3,4)$. Upon activation, platelets also release bioactive mediators such as PDGF and thromboxane, leading to dedifferentiation of VSMCs from a quiescent contractile phenotype to a highly synthetic and proliferating cell type, promoting injury repair (5). Excessive repair, such as observed with intimal hyperplasia in diabetes mellitus (DM), can result from enhanced VSMC dedifferentiation and proliferation (6). The mechanism underlying dysregulation of VSMC phenotypic switching in vascular injury repair in patients with diabetes is unclear. This is clinically important, as diabetic patients are more likely to experience restenosis (intimal hyperplasia) after a vascular procedure $(7,8)$.

\section{Related Commentary: p. 962}

Conflict of interest: The authors have declared that no conflict of interest exists. License: Copyright 2019, American Society for Clinical Investigation.

Submitted: August 27, 2018; Accepted: January 8, 2019.

Reference information: J Clin Invest. 2019;129(3):1372-1386.

https://doi.org/10.1172/JCI124508.
Platelets are anucleate cells without genomic DNA. However, they contain an abundant and diverse array of mRNAs and miRNAs, which appears disproportionately high relative to their low level of protein synthesis (9). Recent advances have demonstrated that APs contain miRNAs in complex with the miRNA effector protein Argonaute 2 (Ago2), which can also be released into the circulation and engaged in horizontal miRNA transfer into other vascular cells, modulating gene expression and function in the recipient cells (10-13). Platelet-derived miRNAs can be internalized by recipient cells including endothelial cells, macrophages, hepatocytes, and tumor cells, and play a role in regulating their functions (14-16). Platelet miRNAs are thus recognized to play important roles in interacting with and modulating many cell types, however, whether platelet miRNAs can modulate VSMCs in vivo, and the pathophysiological consequences of such interactions, is unknown. Such interactions are plausible with arterial injury, denudation of endothelium, and subsequent platelet activation and thrombosis on the exposed vessel wall. The study of platelet-VSMC interactions may provide important insights into arterial injury repair and intimal hyperplasia.

We now demonstrate that internalized APs release key functional miRNAs (miR-143, miR-145, and miR-223) that inhibit expression of their targets, namely Kruppel-like factor 4 (KLF4), KLF5, and PDGFR $\beta$, thereby suppressing VSMC dedifferentiation (promoting resolution of repair). DM platelets exhibited signifi- 
cantly reduced expression of miR-223. Deficiency of miR-223 in platelets under DM conditions and in miR-223-KO mice contributed to enhanced VSMC dedifferentiation, leading to exacerbated intimal hyperplasia. Targeting the mechanisms by which platelet miRNAs regulate the gene expression of VSMCs represents a potential therapeutic strategy for treatment of smooth musclerelated vascular diseases, such as those involving excessive repair, as observed with intimal hyperplasia.

\section{Results}

Platelets are internalized by VSMCs and induce VSMC differentiation. At sites of arterial injury where endothelium is damaged, VSMCs are directly exposed to APs $(1,2)$. We initially cocultured APs (Cell Tracker Green CMFDA-labeled) with human aortic VSMCs. We anticipated that APs would induce VSMC dedifferentiation as part of the recognized wound repair response. Unexpectedly, the protein expression of the VSMC differentiation markers ACTA2, TAGLN, and CNN1 was significantly higher in VSMCs cocultured with APs than those cocultured with resting platelets (RPs) or without platelets (Figure 1A). Concurrent significant decreases in VSMC dedifferentiation markers including KLF4, KLF5, and OPN in VSMCs further supported the induction of a differentiated state by APs (Figure 1A). These effects were also observed at the mRNA level (Supplemental Figure 1; supplemental material available online with this article; https://doi.org/10.1172/JCI124508DS1). VSMCs cocultured with APs also exhibited reduced cell proliferation (Cell Counting Kit-8 [CCK8]), BrdU incorporation (Figure 1, B and C), and expression of PCNA (Supplemental Figure 2), demonstrating decreased proliferation (another hallmark of VSMC differentiation). Taken together, the results suggested that AP coculture promotes, rather than inhibits, VSMC differentiation. This seemed paradoxical, as it is well recognized that platelet activation leads to rapid release of thromboxane and PDGF, both of which reduce VSMC differentiation (repair process). Thus, APs appear to both reduce and promote VSMC differentiation.

Important insights were gained when we cocultured VSMCs with APs for 48 hours and performed confocal microscopy. We observed incorporation of whole APs by VSMCs, and this incorporation progressively increased over time (Figure 1D). Notably, the APs localized to the cytoplasm at early time points (1-4 hours) but to the perinuclear region after 24 hours of coculture (Figure 1D). Confocal laser scanning microscopy with 3D reconstruction supported that APs (green) were internalized into VSMCs, the internalized platelets (green) being on a similar level as the nucleus (blue) (Figure 1E). AP internalization by VSMCs increased with time of coculture and was observed in almost all VSMCs after 24 hours of coculture. Transmission electron microscopy was performed to further confirm the process of platelet internalization in VSMCs. Key to the identification of platelets on electron microscopy is the presence of distinct granules. With APs there was substantial granule release with associated changes in architecture; however, some granules and architecture remained, allowing for precise platelet identification. Initially platelets were at the membrane of VSMCs (Figure 1F, red arrow). This was followed by internalization, where both the internalized VSMC plasma membrane and platelet plasma membrane were intact (Figure 1G, red arrow). Loss of plasma membranes likely occurred through a lysosomal mechanism, followed by incorporation into VSMC cytoplasm (Figure
1, $\mathrm{H}$ and I, red arrow). The sources for each micrograph (lower magnification) are provided in Supplemental Figure 3.

To justify continuing the studies, we wanted to determine whether APs are taken up by injured vessels in vivo. We crossed platelet factor 4-cre ( $\mathrm{PF} 4-\mathrm{cre}$ ) mice (platelet-specific) with the $\mathrm{mT} / \mathrm{mG}$ reporter line (PF4-mT/mG), creating a mouse in which platelets are permanently labeled with mGFP (green), while cells of all other lineages including VSMCs express mTomato (red). Thus, red VSMCs would turn green from incorporation of mGFP if platelets were incorporated. Femoral artery wire injury was performed to damage endothelium and activate platelets (an in vivo model for induction of VSMC dedifferentiation and repair). No platelets (green) were detected in the uninjured femoral arteries (Figure 1J). However, 1 week after injury, mGFP-positive cells were readily found in the medial VSMC layer of the injured vessel (Figure 1J, arrows). Importantly, we demonstrated that the expression of cre recombinase can be detected in both resting and activated platelets isolated from $\mathrm{PF} 4-\mathrm{mT} / \mathrm{mG}$ mice, but not WT mice (Supplemental Figure 4). Platelets (no genomic DNA) and VSMCs were also isolated from PF4-mT/mG mice and cocultured for 48 hours. We detected genomic DNA recombination from mouse VSMCs cocultured (48 hours) with APs and the cre groups in comparison to the RP group (Supplemental Figure 5). Based on these initial in vitro and in vivo data, we developed the hypothesis that upon arterial injury, APs are internalized by VSMCs (hours to days) at sites of injury and may serve as a VSMC phenotypic switch.

APs transfer miRNAs into VSMCs to inhibit VSMC dedifferentiation. Incorporated platelets must contain potent agents to initiate the switch from VSMC dedifferentiation to differentiation. Platelets have been reported to contain a large number of miRNAs (1719). To initially determine whether platelet internalization could transfer miRNA, we used a megakaryoblastic cell line, MEG-01, to produce labeled platelets (15). Platelets were incorporated with (i) labeled RNA (assessed by 5-ethynyluridine [EU] incorporation) (Supplemental Figure 6A), (ii) transfected synthetic exogenous miRNA syn-cel-miR-39 (Supplemental Figure 6B), and (iii) transfected FITC-labeled scrambled miRNA (miR-Scr-FITC) (Supplemental Figure 6C). Each demonstrated incorporation of platelet-derived miRNAs in VSMCs. Based on the electron microscopy (Figure 1F and Supplemental Figure 3), we hypothesize that the miRNA is initially located in the APs but is released upon internalization and removal of the platelet cell membrane. Taken together, these in vitro results supported transfer of miRNA from incorporated APs into VSMCs.

To identify platelet miRNAs that may be involved in the horizontal transfer and inhibit VSMC dedifferentiation, we performed miRNA-Seq on VSMCs cocultured with or without APs (control) (Figure 2A). We identified a number of miRNAs that were differentially expressed in VSMCs when cocultured with APs, including key miRNAs with established roles in regulating VSMC phenotype, such as miR-223, as well as the VSMC master miRNA cluster miR-143/145. These changes were independently validated by quantitative PCR (qPCR) at various time points $(2,4,8,24$, and 48 hours) following coculture with APs. We found significant upregulation of miRNA expression in the AP compared with the control group, with expression peaking at 4 hours for miR-223 or 24 hours for miR-143/145 (Figure 2B). Since miRNAs are known to incorpo- 
A

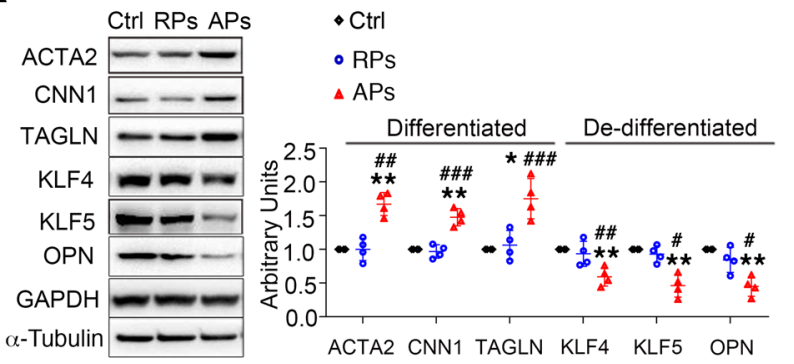

B

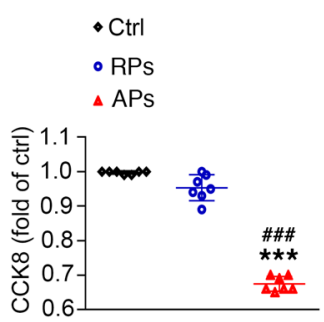

C

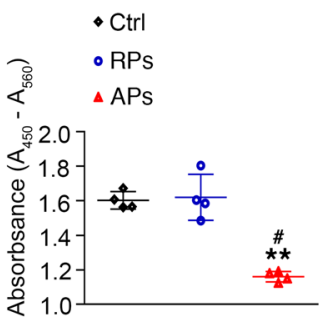

D

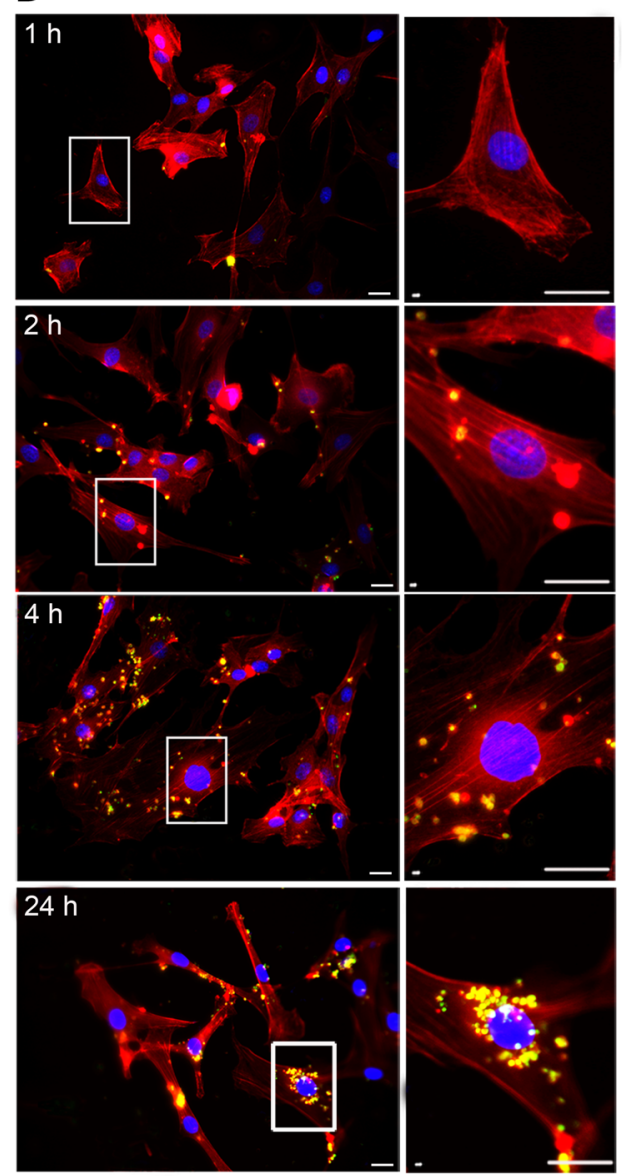

Platelet / ACTA2 / Hoechst
E
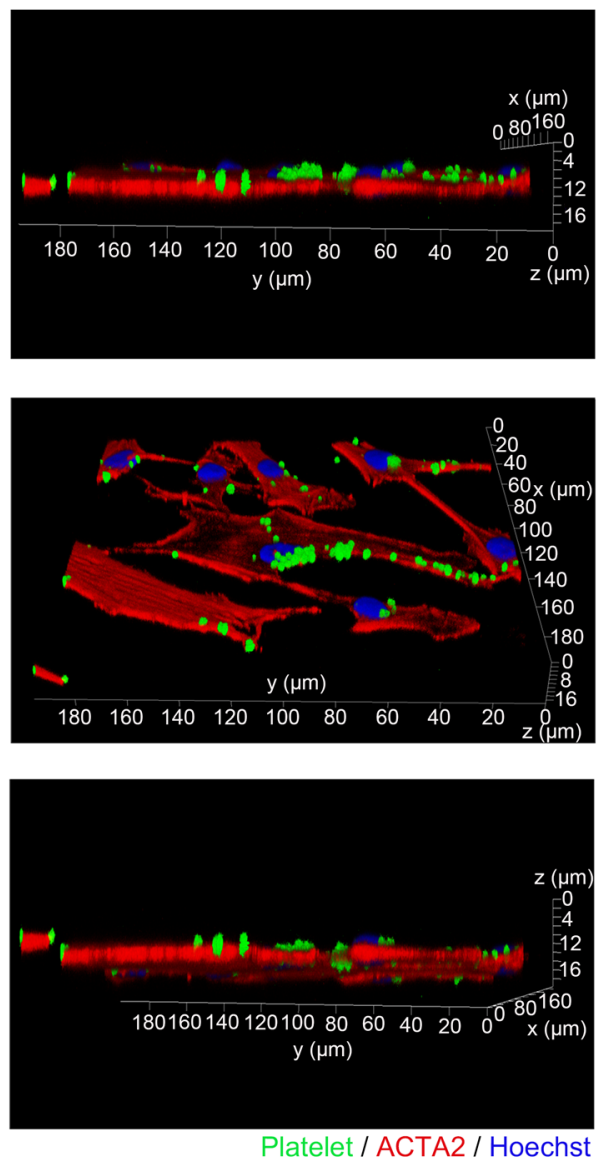

H

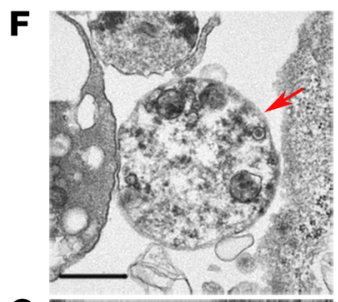

G

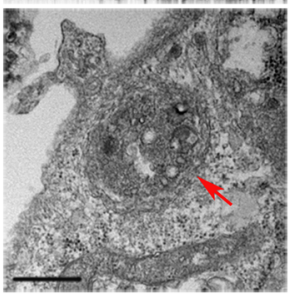

H

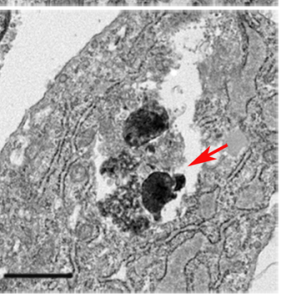

I

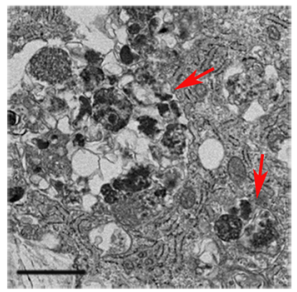

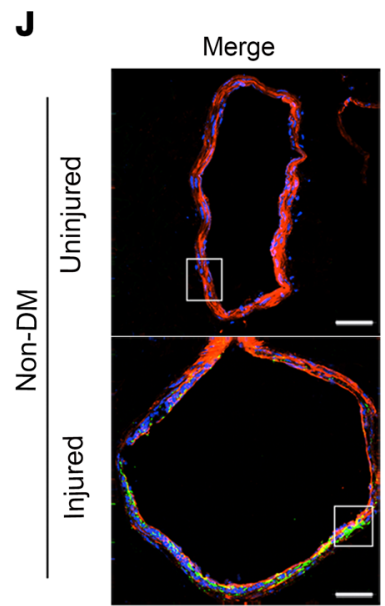

mTomato

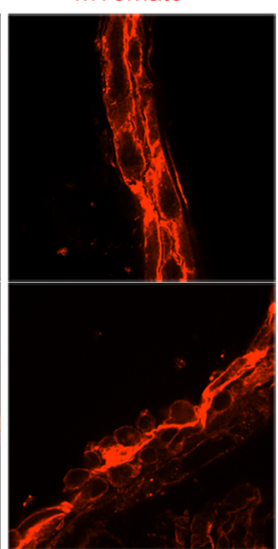

mGFP

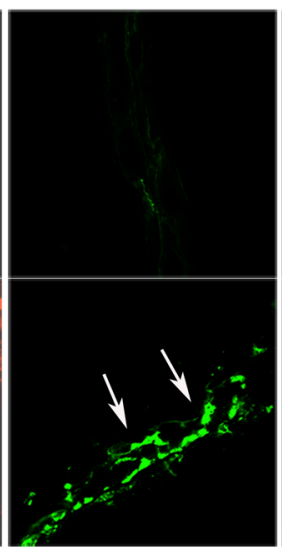

DAPI

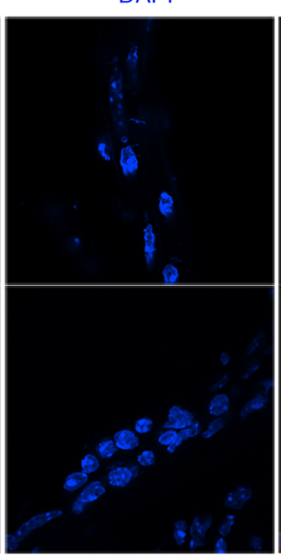

Merge

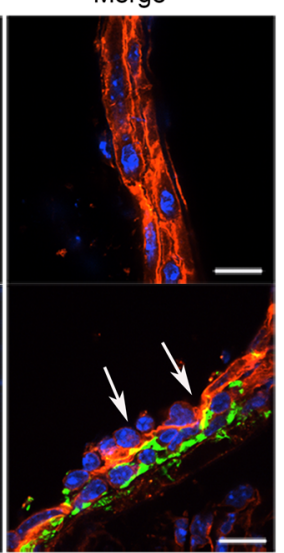


Figure 1. Platelets were internalized by VSMCs and induced VSMC differentiation. (A) Expression of markers for differentiation (ACTA2, CNN1, TAGLN) and dedifferentiation (KLF4, KLF5, OPN) in control VSMCs (Ctrl) and in VSMCs after coculture with RPs or APs was determined by Western blot analysis $(n=4)$. Cell proliferation was assessed by CCK8 assays $(n=7)$ $(B)$ and BrdU incorporation assays $(n=4)(C)$. Data are presented as mean \pm SD. ${ }^{*} P<0.05,{ }^{* *} P<0.01,{ }^{* *} P<0.001$ vs. Ctrl; ${ }^{\#} P<0.05,{ }^{\# \#} P<0.01$, $\# \# P<0.001$ vs. RPs. (D) Representative images of VSMCs cocultured with CMFDA-labeled platelets (green) for 1, 2, 4, or 24 hours in the presence of thrombin. VSMCs were stained with ACTA2 (red) and nuclei visualized with DAPI $(n=6)$. Scale bars: $2.5 \mu \mathrm{m}$. (E) $3 D$ reconstruction of confocal $Z$-stack images of the whole VSMCs cocultured with CMFDA-labeled platelets (green). (F-I) Transmission electron microscopy imaging of VSMCs cocultured with APs. Panels provide sequence of platelet internalization and incorporation into VSMCs. The red arrow indicates the entering and internalized platelet. Lower-magnification source electron micrographs are shown in Supplemental Figure 3. Scale bars: $1 \mu \mathrm{m}$. (J) Representative images of sections from uninjured and injured femoral arteries of PF4-mT/ $\mathrm{mG}$ and WT mice $(n=7)$. Arrows indicated multiple VSMCs with incorporated green platelets. The injured femoral arteries were harvested on the seventh day after injury. Scale bars: $20 \mu \mathrm{m}$. Statistical significance was determined using 1-way ANOVA followed by Tukey-Kramer multiplecomparisons test $(\mathbf{A}-\mathbf{C})$.

rate into Argonaute 2 (Ago2) effector complexes for the regulation of specific mRNAs through translational repression, we performed Ago2 immunoprecipitation, followed by qPCR detection of miR223 and miR-143/145. Our results demonstrated the presence of Ago2·miR-143, Ago2·miR-145, and Ago2·miR-223 complexes in platelets, suggesting that the increased miRNAs were functional in VSMCs after coculturing with APs (Supplemental Figure 7). Pretreatment with prostacyclin (which prevents platelet activation) or RNase A (degrades the RNA) in platelets significantly attenuated miR-223 and miR-143/145 in VSMCs (Figure 2C), suggesting transfer of miRNA rather than induction in VSMCs; the finding is consistent with previous observations in endothelial cells and hepatocytes $(14,15)$. Importantly, pretreatment with these reagents (prostacyclin and RNase A) significantly reduced the prodifferentiation effect induced by APs on VSMCs (Figure 2D). The observed changes were not due to an effect of thrombin itself (used to activate the platelets) on the endogenous expression of these 3 miRNAs in VSMCs (Supplemental Figure 8). Taken together, these results support that the uptake of APs by VSMCs leads to horizontal transfer of platelet miRNA into VSMCs, resulting in a switch from VSMC dedifferentiation to VSMC differentiation.

$K L F 4, K L F 5$, and PDGFR $\beta$ are the critical targets of miR-143, miR-145, and miR-223 for regulating VSMC dedifferentiation. Bioinformatics analysis identified a conserved binding site for miR-145 in the 3'-UTR of KLF4. For miR-143 and miR-145, putative binding sites were located at the 3 '-UTR of KLF5, and for miR-143 and miR-223 putative sites at the 3'-UTR of PDGFR $\beta$ (Supplemental Figure 9). To determine whether miR-143 and miR-223 could bind to PDGFR $\beta$ and inhibit its expression directly, we cloned a fragment of the $3^{\prime}$-UTR of PDGFR $\beta$ into a luciferase reporter. Adding either a miR-143 mimic or miR-223 mimic (Supplemental Figure 10) resulted in significant inhibition of luciferase activity, while in the mutated control groups the inhibitory effect of miR-143 mimic and miR-223 mimic on luciferase activity was abrogated (Supplemental Figure 11, A and B). Our data demonstrate for the first time to our knowledge that miR-143 and miR-223 can directly target the $3^{\prime}$-UTR of PDGFR $\beta$ (Supplemental Figure 11, A and B) and inhibit its expression (Supplemental Figure 11C). Similarly, miR-143 could directly target the 3'-UTR of KLF5 (Supplemental Figure 11D) and inhibit its expression (Supplemental Figure $11 \mathrm{~F})$, This observation is supported by previous studies $(20,21)$. Our results also showed that miR-145 can directly bind to the 3'-UTR of KLF4 and KLF5 (Supplemental Figure 11, E and G) and inhibit expression (Supplemental Figure 11, F and H), which is also supported by previous reports (22). Taken together, these results indicate that miR-143, miR-145, and miR-223 can regulate VSMC dedifferentiation by targeting KLF4, KLF5, and PDGFR $\beta$, respectively, in VSMCs. Therefore, horizontal transfer of those miRNAs from APs to VSMCs may contribute to VSMC phenotypic switching.

In order to further demonstrate that externally derived (platelet) miR-143, miR-145, and miR-223 are indeed critical to VSMC phenotypic switching, rescue experiments were needed. We found that expression of miR-143, miR-145, and miR-223 was significantly reduced in platelet-like particles (PLPs) derived from MEG-01 cells under conditions of high glucose (HG; $33 \mathrm{mM}$ ) compared with the levels of glucose found in the normal growth media (NG; $17.5 \mathrm{mM}$ ) (Figure 3A). To verify the importance of platelet-derived miRNAs in regulating VSMC phenotype, we cocultured VSMCs with PLPs derived from MEG-01 under normal glycemic (NG PLPs) or high-glucose (HG PLPs) conditions. As expected, NG PLPs significantly inhibited the expression of PDGFR $\beta$, KLF4, and KLF5 (Figure 3B). However, HG PLPs with reduced levels of miRNAs had no effect on regulation of expression of these 3 proteins (Figure 3B). Next, Lipofectamine transfection was performed to restore the expression levels of miR-143, miR-145, and miR-223 in HG PLPs (miRNA PLPs) (Supplemental Figure 12). We found that expression of PDGFR $\beta$, KLF4, and KLF5 was significantly inhibited in miRNA PLPs, resulting in VSMC differentiation (Figure 3C). Moreover, we found that the inhibitory effect of miR-223 was delayed compared with PDGF stimulation in vitro. At early time points (6-12 hours) of coculture, PDGF released from platelets promoted VSMC proliferation, and platelet-derived miR-223 accumulated in VSMCs (Figure 3D). At the later time points (24-48 hours), the platelet-derived miR-223 was utilized by VSMCs, resulting in inhibition of cell proliferation and expression of its target, PDGFR $\beta$ (Figure 3D). Taken together, these data demonstrate that miRNAs released by platelets are a major regulator of VSMC phenotype switch.

miR-223 modulates Pdgfr $\beta$ expression and intimal hyperplasia in vivo after femoral arterial injury. miR-143 and miR-145 are native to both platelets and VSMCs, whereas miR-223 is specifically expressed in the hematopoietic system (23) (including platelets), with no significant VSMC expression. As proof of principle for our hypothesis that uptake of APs by VSMCs transfers miRNA that leads to a phenotypic switch, we used platelet miR-223 KO mice and rescued the condition with AgomiR-223 through local delivery at the site of femoral wire injury. We first determined the expression levels of Pdgfr $\beta$ (dedifferentiation) and Acta2 (differentiation) in femoral arteries with or without injury. As shown in Figure 4A, in uninjured vessels, expression of Pdgfr $\beta$ was very low. At 4 weeks after injury, expression of Pdgfr $\beta$ was upregulated par- 
A
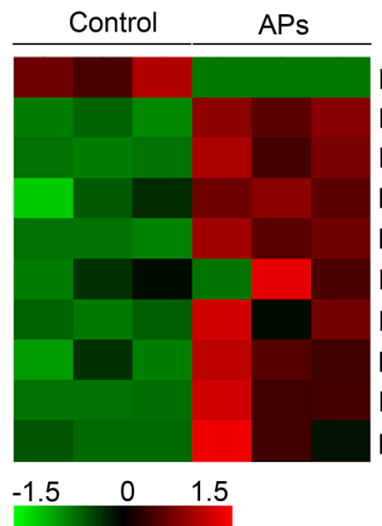

B
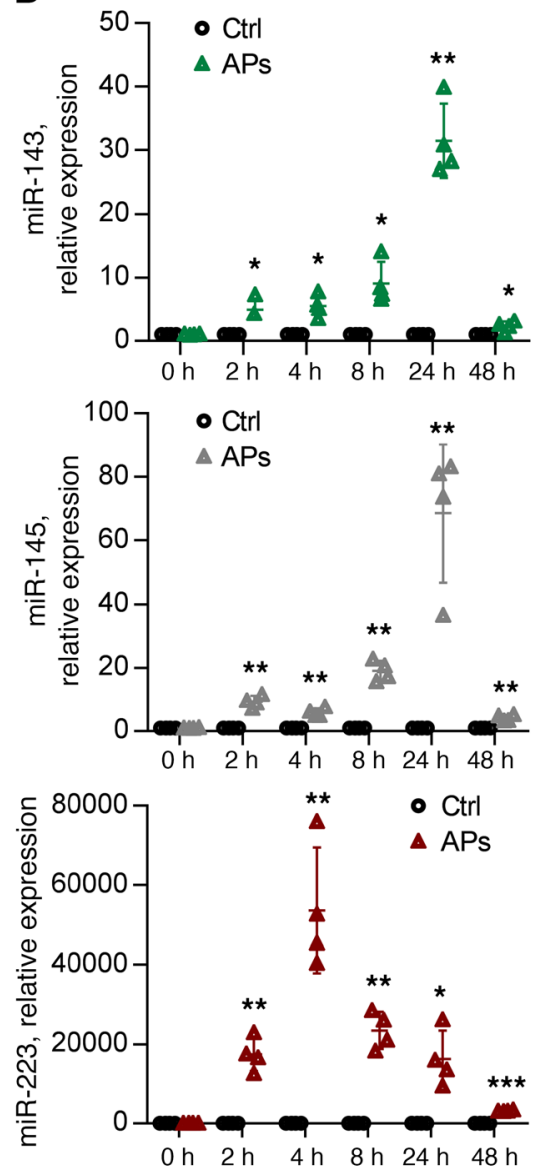

C

hsa-miR-1323

hsa-miR-142-3p

hsa-miR-142-5p

hsa-miR-143-3p

hsa-miR-144-3p

hsa-miR-145-5p

hsa-miR-146a-5p

hsa-miR-200a-3p

hsa-miR-223-3p

hsa-miR-3126-3p

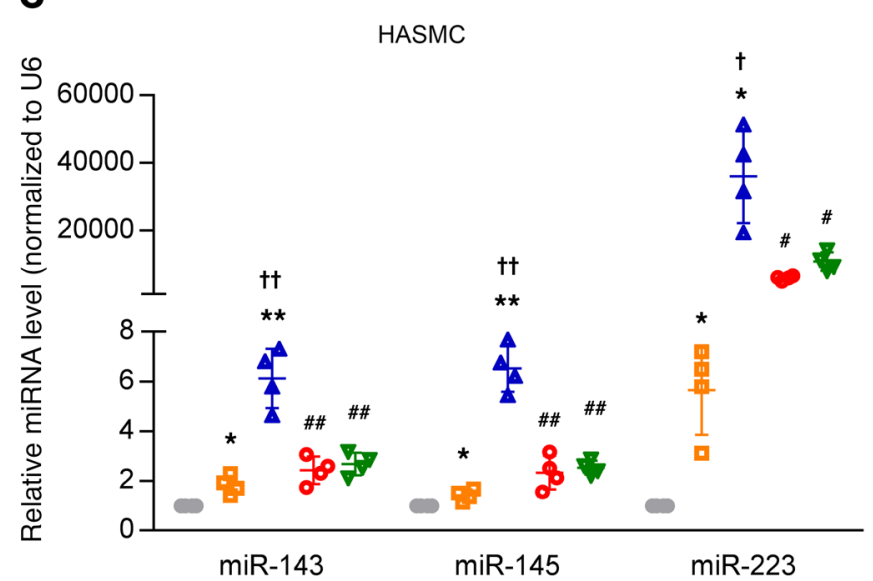

D
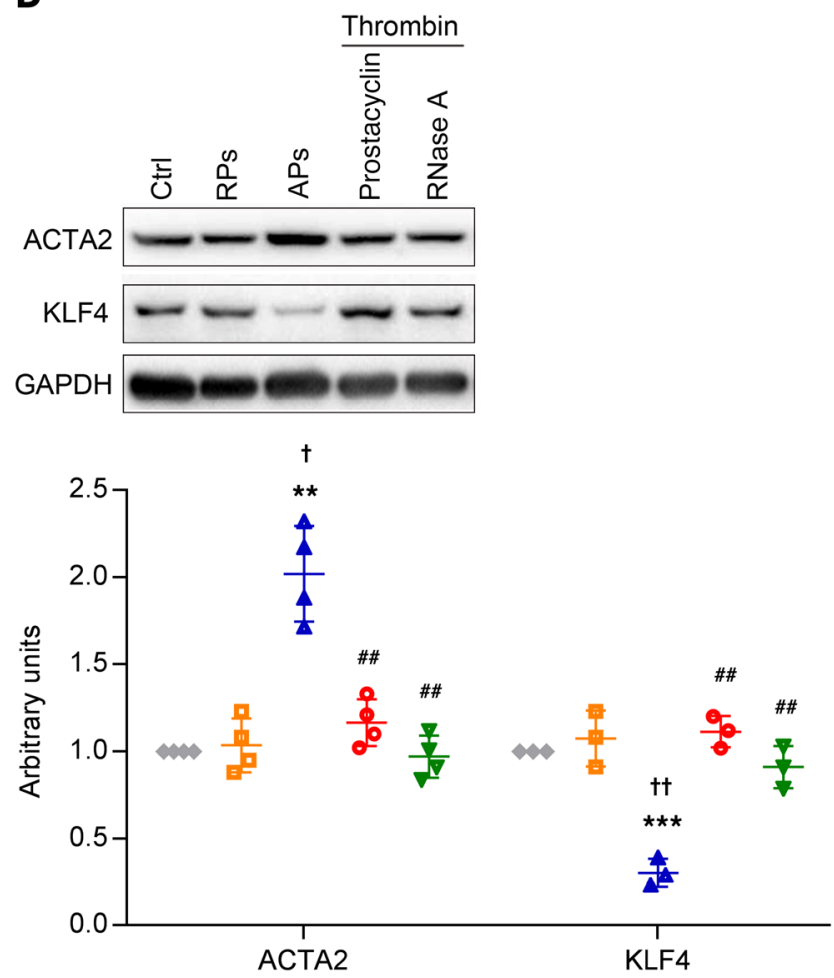

- Ctrl

a RPs

$\triangle \mathrm{APs}$

- Prostacyclin + thrombin

$\checkmark$ RNAse A + thrombin
- Ctrl

口 RPs

$\triangle$ APs

- Prostacyclin + thrombin

$\nabla$ RNAse A+ thrombin

Figure 2. APs transferred miR-143, miR-145, and miR-223 into VSMCs, and promoted VSMC differentiation. (A) Heatmap of the top 10 differentially expressed miRNAs in VSMCs cocultured with or without APs for 24 hours $(n=3)$. (B) Expression of miR-143, miR-145, and miR-223 in VSMCs (Ctrl) and VSMCs cocultured with APs $(n=4)$. (C) Expression of miR-143, miR-145, and miR-223 in VSMCs $(C t r l)$ and VSMCs cocultured with RPs or APs after pretreatment with prostacyclin or RNase A $(n=4)$. (D) The expression of ACTA2 and KLF4 in control VSMCs (Ctrl) and VSMCs cocultured with RPs or APs after pretreatment with prostacyclin or RNase A and thrombin $(n=4)$. Data are presented as mean \pm SD. ${ }^{*} P<0.05$, ${ }^{* *} P<0.01$, ***P $P<0.001$ vs. Ctrl; ${ }^{\dagger} P<0.05,{ }^{\dagger} P<0.01$ vs. RPs; ${ }^{\#} P<0.05,{ }^{\# \# P}<0.01$ vs. APs. Statistical significance was determined using parametric $t$ test (B) and $1-$ way ANOVA followed by Tukey-Kramer multiple-comparisons test (C and D).

ticularly in the miR-223-KO mice treated with miR negative control (miR-NC) (Figure 4, A and B). Treatment with AgomiR-223 significantly rescued the effect, decreasing expression of Pdgfr $\beta$ in miR-223 KO mice (Figure 4, A and B). These results suggest that Pdgfr $\beta$ is indeed a target gene of miR-223 in vivo. Pheno- typic change in VSMCs plays a critical role in vascular neointimal development (intimal hyperplasia). We thus determined the effect of miR-223 on neointimal growth. After 4 weeks of femoral arterial injury, increased intimal size was observed in the miR-223-KO mice treated with AgomiR-NC (Figure 4C), as 
A
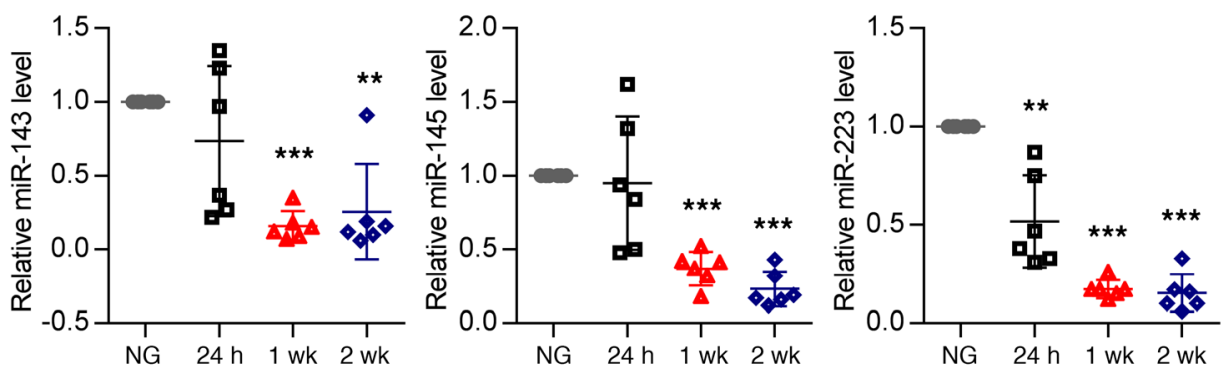

B
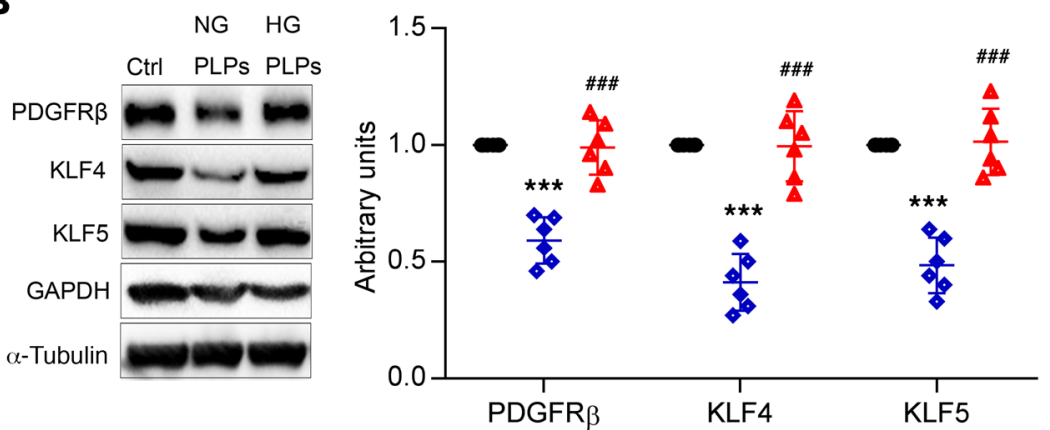

C
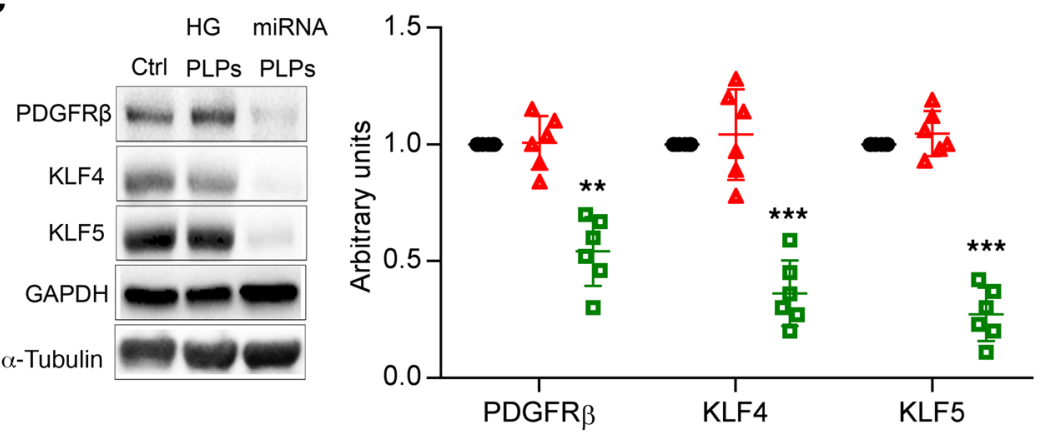

- Ctrl

$\triangle$ HGPLPS

口 miRNAPLPs

Figure 3. Incorporation of platelet-derived miRNAs is required for inhibiting VSMC dedifferentiation. (A) Levels of miR-143/145 and miR223 in PLPs derived from MEG-01 cells under normal glucose (NG; 17.5 $\mathrm{mM}$ ) or high glucose (HG; $35 \mathrm{mM})$ for 0 hours, 24 hours, 1 week, or 2 weeks. Data are presented as mean $\pm \mathrm{SD}(n=6) .{ }^{* *} P<0.01,{ }^{* * *} P<0.001$ vs. NG. (B) Expression of PDGFR $\beta$, KLF4, and KLF5 in VSMCs cocultured with or without PLPs derived from MEG-01 cells under NG (NG PLPs) or HG (HG PLPs). Data are presented as mean $\pm \operatorname{SD}(n=6) .{ }^{* *} P<0.001$

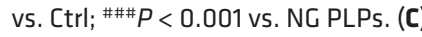
Expression of PDGFR $\beta$, KLF4, and KLF5 in VSMCs cocultured with or without PLPs derived from MEG-01 cells under HG (HG PLPs), or HG PLPs transfected with miR-143, miR-145, and miR-223 mimics (miRNA PLPs). Data are presented as mean \pm SD $(n=6) .{ }^{* *} P<0.01,{ }^{* * *} P<0.001$ vs HG PLPs. (D) VSMCs were cocultured with APs for 3, 6, 12, 24, and 48 hours. PDGFR $\beta$ expression (red line), cell proliferation rate (blue line), and the miR-223 level (black line) were detected by Western blot, BrdU incorporation assay, and qPCR, respectively. Data are presented as mean \pm SD $(n=4) .{ }^{* *} P<0.01,{ }^{* *} P$ $<0.001$ vs 3h. Statistical significance was determined using 1-way ANOVA followed by Tukey-Kramer multiple-comparisons test (A-D).

D

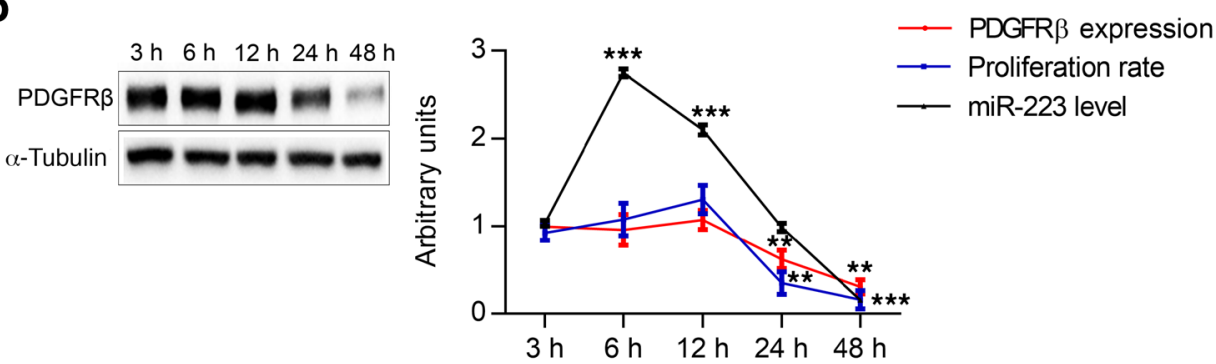

indicated by the significantly increased intima to media (I/M) ratio (Figure 4D). Restoration of the downregulated miR-223 in wire-injured femoral arteries significantly inhibited neointimal hyperplasia (Figure 4, C and D). An additional experiment demonstrated that the levels of internalized miR-223 in VSMCs cocultured with whole APs were significantly higher than in those cocultured with AP-derived microparticles (Supplemental Figure 13). Along with the size and morphology of the internalized platelets (Figure $1 \mathrm{~F}$ ), the results support whole platelet uptake rather than internalization of platelet-derived microparticles. Taken together, these studies support a phenotypic switch provided by the transfer of miR-223 from activated whole platelets to VSMCs.
$D M$ mice have reduced miR-223 and increased intimal hyperplasia. It has been reported that the levels of miRNAs in platelets are altered under pathological conditions, such as in DM $(24,25)$. Assessment of both human and mouse DM platelets demonstrated consistent reductions in miR-223 expression (Figure 5A). Femoral wire injury experiments in DM mice demonstrated substantial incorporation of platelets (green) into VSMCs in the media, as previously observed with WT mice (Figure 5B). Given that levels of miR-223 are reduced in DM platelets (Figure 5A), we set out to determine VSMC injury response, comparing DM with WT mice. We initially performed FISH in femoral wire-injured arteries to directly detect and visualize miR-223 uptake in VSMCs. One week after wire injury, levels 
A

Uninjured

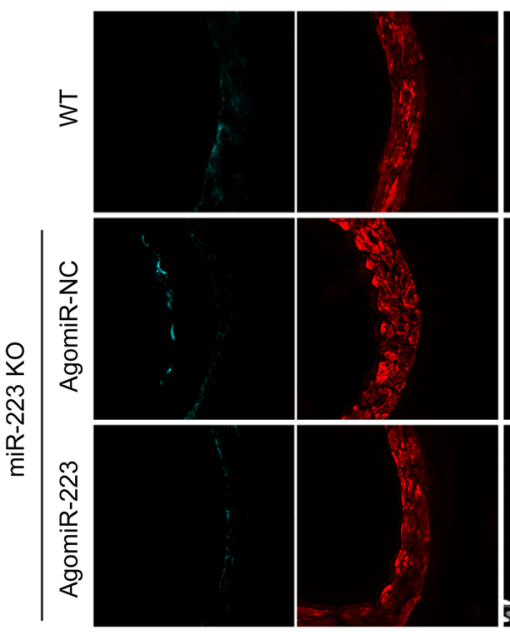

B

Pdgfr $\beta$

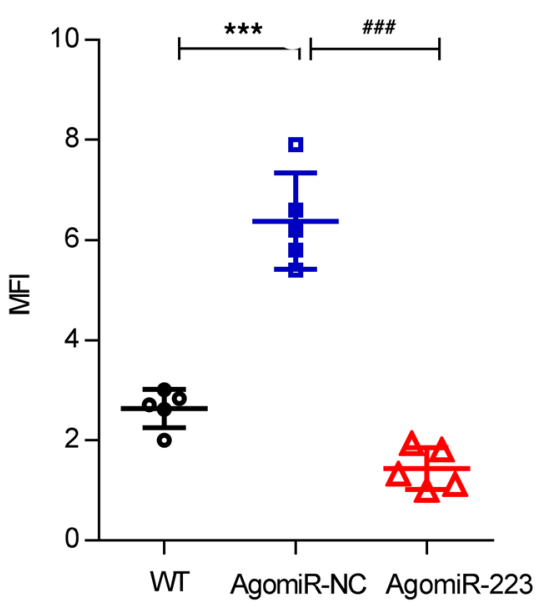

$\operatorname{miR}-223 \mathrm{KO}$

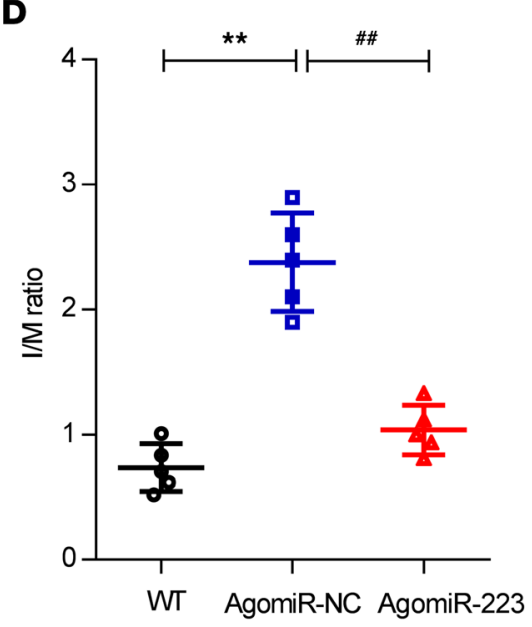

miR-223 KO
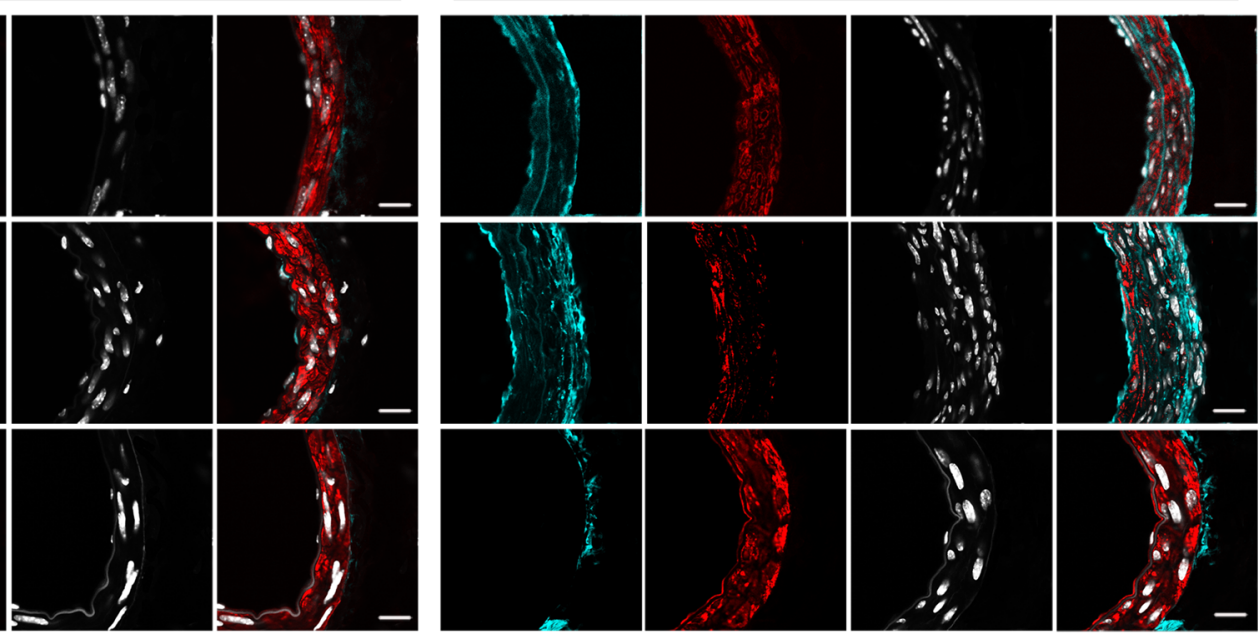

Pdgfr / Acta2 / DAP

C
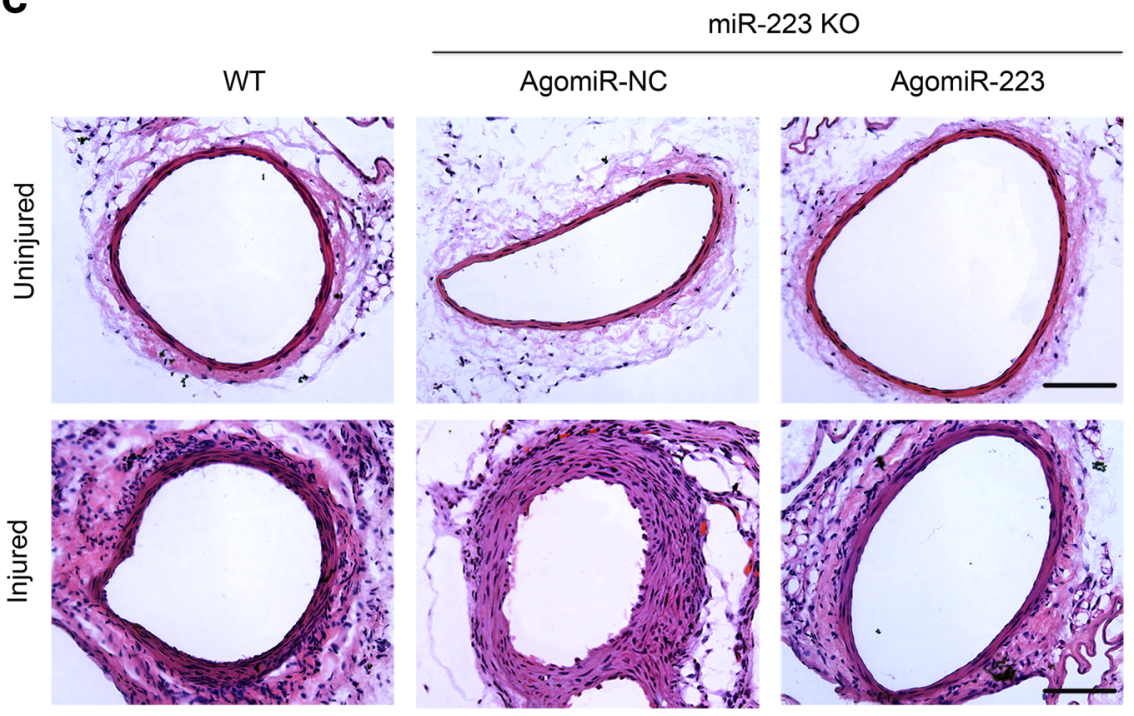

Figure 4. $\mathrm{miR}-223$ modulates Pdgfr $\beta$ expression and intimal hyperplasia in vivo after femoral arterial injury. (A) Representative immunofluorescence of Pdgfr $\beta$ in uninjured or injured femoral arteries from WT mice, and miR-223 KO mice treated with AgomiR-NC or AgomiR-223 at 4 weeks after wire injury $(n=5)$. Green, Pdgfr $\beta$; blue, DAPI nuclear staining; red, Acta2 in VSMCs. Scale bars: $20 \mu \mathrm{m}$. (B) Quantification of Pdgfr $\beta$ expression in VSMCs in injured femoral arteries. Data are presented as mean \pm SD $(n=5) .{ }^{* *} P<0.001$ vs. WT; ${ }^{\# \#} P<0.001$ vs. miR-223 KO mice treated with AgomiR-NC. (C) H\&E staining of serial cross sections of femoral arteries from WT mice, and miR-223 KO mice treated with AgomiR-NC or AgomiR-223 at 4 weeks after injury $(n=5)$. Scale bars: $100 \mu \mathrm{m}$. (D) Morphometric measurements of the I/M ratio in the injured femoral arterial sections. Data are presented as mean \pm SD of I/M ratio $(n=5)$. ${ }^{* *} P<0.01$ vs. WT; ${ }^{\# \#} P<0.01$ vs. miR-223 KO mice treated with AgomiR-NC. Statistical significance was determined using 1-way ANOVA followed by Tukey-Kramer multiple-comparisons test (B and $\mathbf{D})$. 
A
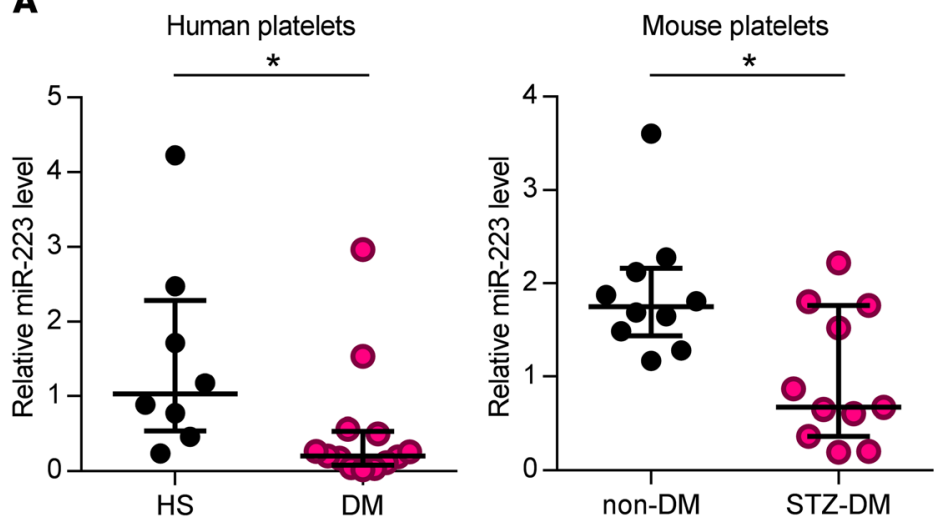

B

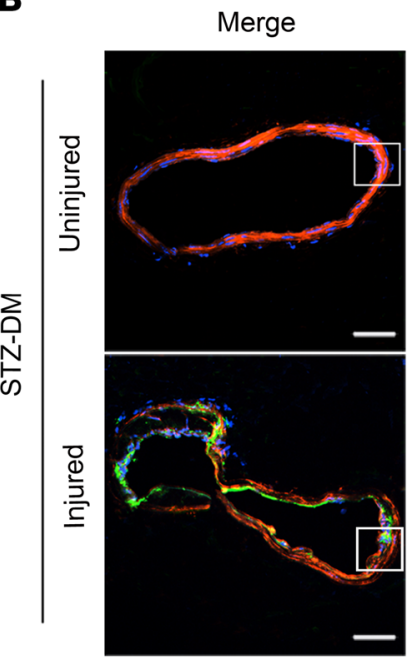

mTomato

mGFP
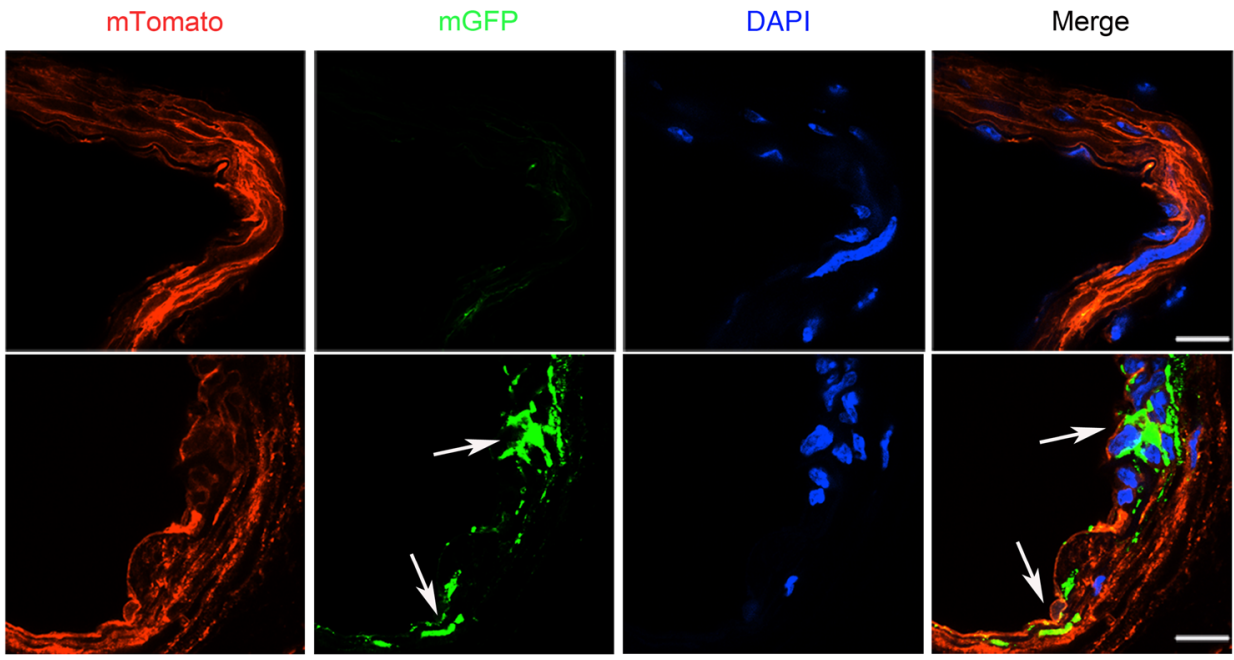

C

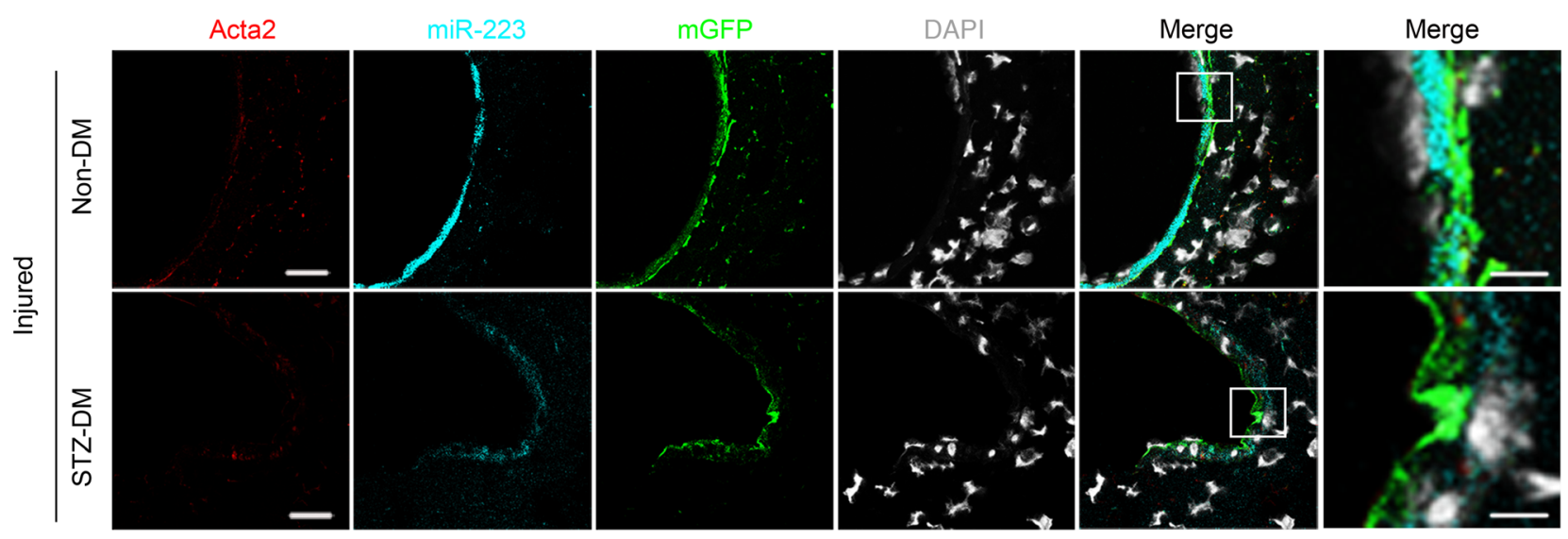

D

$\operatorname{miR}-223$

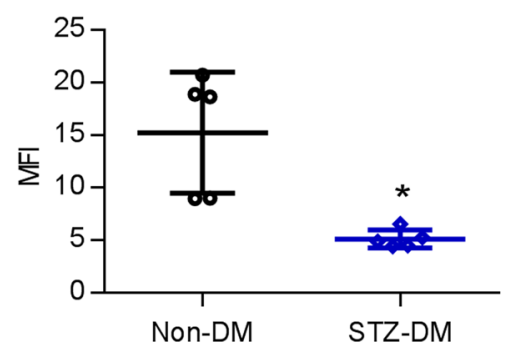

Figure 5. The miR-223 level was reduced in DM platelets and VSMCs in injured femoral arteries. (A) Expression of miR-223 in platelets from healthy subjects (HS, $n=8$ ) and DM patients (DM, $n=13$ ); and non-DM murine (non-DM, $n=10)$ and STZ-DM mice $(n=11)$. Data are presented as medians and IQRs. ${ }^{*} P<0.05$ vs. HS or non-DM. (B) Representative images of the sections from uninjured and injured femoral arteries of PF4-cre:mT/mG STZ-DM mice $(n=7)$. Arrows indicate VSMCs with incorporated platelets. The injured femoral arteries were harvested on the seventh day after injury. Scale bars: $20 \mu \mathrm{m}$. (C) Representative images of miR-223 expression in injured femoral arteries of PF4-cre:mT/mG mice under non-DM or STZ-DM conditions $(n=5)$. The injured femoral arteries were harvested on the seventh day after injury. Red, Acta2; green, mGFP; white, DAPI. Scale bars: $20 \mu \mathrm{m}$. (D) Quantification of the expression of miR-223 in mGFP-positive VSMCs in the injured femoral arteries $(n=5)$. Data are presented as mean $\pm \mathrm{SD}$. ${ }^{*} P<0.05$ vs. non-DM. Statistical significance was determined using Mann-Whitney $U$ test (A) and parametric $t$ test (D). 
of miR-223 in mGFP-positive VSMCs (contributed by APs) were notably higher in non-DM mice than in mice with streptozotocininduced (STZ-induced) DM (Figure 5C). Quantitation using ImageJ demonstrated a significant $(P<0.01)$ reduction in the STZ-DM versus non-DM mice (Figure 5D). These in vivo results are supported by the in vitro mechanistic studies and are consistent with the low miRNA levels observed in mouse STZ-DM platelets and human DM platelets. Moreover, concurrent staining for Acta2 was increased in the non-DM compared with STZ-DM (lower miRNAs) mice, also supporting the in vitro studies. Thus, under STZ-DM conditions, although platelets were incorporated into medial VSMCs, there was significantly reduced horizontal transfer of platelet miRNAs. These results provide the first direct visualization to our knowledge of platelet-derived miRNA in VSMCs. However, although reduced transfer of miRNA is supported by the reduced Acta2 staining, further proof was needed to demonstrate such incorporation has a biochemical and biological effect on VSMCs and injury repair.

We next set out to determine (using high-resolution confocal microscopy) whether this translated to increased expression of the miRNAs' target genes in vivo (in single VSMCs) that have taken up the APs (green VSMCs). Basal expression of Pdgfr $\beta$ was undetectable by immunofluorescence in femoral arteries of non-DM and STZ-DM mice (Figure 6A). As expected, 1 week after wire injury, Pdgfr $\beta$ expression was increased in the non-DM group, but more so in the STZ-DM group (Figure 6A), reflecting the dedifferentiated state. We detected 2 populations of VSMCs in the media of injured tissues - VSMCs with internalized platelets (green with DAPI) and those without (DAPI alone) (Figure 6A). Media VSMCs with internalized platelets demonstrated significantly increased Pdgfr $\beta$ under STZ-DM vs. non-DM conditions, reflecting reduced miR-223 in DM platelets (Figure 6B). VSMCs with internalized platelets also exhibited increased expression of Acta 2 with reduced expression of Pdgfr $\beta$ (Figure 6, A and B). These results are consistent with our in vitro findings that horizontal transfer of plateletderived miR-223 into VSMCs inhibits VSMC dedifferentiation via downregulation of Pdgfr $\beta$. In the absence of miR-223, such as under STZ-DM conditions or in miR-223 KO, the expression of $\operatorname{Pdgfr} \beta$ was increased in the injured vessels, contributing to VSMC proliferation and intimal hyperplasia.

We then used miR-223 platelet $\mathrm{KO}$ and rescue to assess whether DM leads to excess intimal hyperplasia (excess injury repair) and look for the degree of rescue with AgomiR-223. In uninjured vessels, expression of Acta2 was consistently high and Pdgfr $\beta$ was very low (Figure 6, C and D). At 4 weeks after injury, expression of Acta2 was very low in the STZ-DM vessels treated with control and appeared to be rescued with AgomiR-223 (Figure 6, C and D). The converse was true, with Pdgfr $\beta$, supporting the targeting of Pdgfr $\beta$ by miR-223. Moreover, when we assessed for intimal hyperplasia, DM arteries demonstrated a doubling of the I/M ratio, which was in part rescued by AgomiR-223 (Figure 6, E and F). Taken together these studies support a phenotypic switch provided by the transfer of miR-223 from APs to VSMCs. In $\mathrm{DM}$, the injury repair response was increased, and could be reduced by miR-223 mimics.

Model for platelet-VSMC interaction. During normal physiology, the endothelial barrier is intact and platelets are quiescent, exhibiting no interactions with VSMCs (Figure 7). With injury (as with angioplasty), the endothelial barrier is compromised, exposing the subendothelium, leading to platelet activation. In addition to thrombosis, there is release of agents (immediate) that initiate wound repair, including induction of VSMC dedifferentiation and proliferation. APs are then taken up by VSMCs (hours to days), releasing miR-223 (and others, such as miR-143/145), which applies a brake on VSMC dedifferentiation, switching to VSMC differentiation. This second "wave" (delayed response) prevents excess VSMC repair by promoting injury response resolution and thus reduced intimal hyperplasia. With reduced miR-223 in DM platelets, excess VSMC proliferation (reduced differentiation) leads to excess intimal hyperplasia.

\section{Discussion}

Upon vascular injury, the initial response is to maintain hemostasis with clot formation, vessel contraction, and cellular proliferation. This immediate response is mediated largely by substances such as thromboxane and PDGF, which are released from APs, promoting VSMC dedifferentiation in wound healing (26-28). If the response is left unabated, substantial intimal hyperplasia can occur. To prevent severe intimal hyperplasia, a brake needs to be applied relatively early to regulate the VSMC proliferative response (Figure 6).

Direct visualization of platelet uptake. We report that APs are directly incorporated into VSMCs at sites of injury to apply this needed brake. Our femoral artery wire injury model allowed for direct in vivo visualization of the interaction between circulating platelets and exposed VSMCs due to the denudation of the endothelium layer. To our knowledge, this is the first study to establish an in vivo model directly visualizing internalization of platelets (expressing $\mathrm{PF} 4$-icre driving $\mathrm{mT} / \mathrm{mG}$ recombination) into recipient cells. In this double-fluorescence system, mTomato-labeled VSMCs (red) became mGFP-positive (green) once incorporated into platelets. Individual recipient VSMCs could be precisely located in the injured artery, and biochemical changes followed. High expression of PDGFR $\beta$ in VSMCs exemplifies VSMC dedifferentiation, contributing to VSMC proliferation and intimal hyperplasia (29). mGFP-expressing VSMCs (uptake of platelets) showed reduced expression of PDGFR $\beta$ compared with mTomatoexpressing VSMCs (no platelet uptake), suggesting that platelet internalization can suppress VSMC dedifferentiation. We hypothesize that platelet internalization is likely followed by lysis of the platelet cell membrane, allowing for release of contents such as miRNA. Taken together, our results provide direct visual and biochemical evidence that platelets orchestrate the VSMC response at the site of vascular injury.

Identification of critical platelet-derived miRNAs that regulate the VSMC switch. Using genome-wide miRNA screening, we identified key platelet miRNAs (miR-223 and miR143/145) that target VSMC differentiation and proliferation genes. Platelet-derived miRNAs are ideal, as they can catalytically regulate many proteins and coordinately ensure return to a differentiated quiescent state. We demonstrate for the first time that platelet-derived miR-223 can downregulate PDGFR $\beta$ in VSMCs, in turn inhibiting PDGFinduced VSMC proliferation. Previous studies have demonstrated that PDGFR $\beta$ is primarily expressed in VSMCs (30), and inhibi- 
tion of PDGFR $\beta$ suppressed VSMC proliferation in vitro and in the balloon-injured artery model (31). In addition, we also found that the platelet-derived miR-143 and miR-145 can downregulate KLF4 in VSMCs, corroborating previous studies showing that endogenous VSMC miR-143 and miR-145 induces differentiation by directly targeting KLF4 and KLF5 (22, 32). KLF4 and KLF5 are the master regulators of VSMC dedifferentiation, and inhibition of KLF4 and KLF5 expression promotes VSMC differentiation. Taken together, our results demonstrate, both in vitro and in vivo, that platelet-derived miR-223 is incorporated into VSMCs and directly target, PDGFR $\beta$, respectively, resulting in inhibition of VSMC dedifferentiation and reduction of intimal hyperplasia. Thus, VSMC response to injury represents a delicate balance between early dedifferentiation and late differentiation during recovery.

Diabetes and intimal hyperplasia. Key to our studies is the observation that DM platelets have reduced miR-223 expression and thus a reduced ability to apply a brake on VSMC dedifferentiation. DM patients are recognized to be more prone to restenosis (intimal hyperplasia) and less responsive to drug-eluting stent agents (33). We induced diabetes mellitus in PF4-mT/mG mice and performed femoral artery wire injury. Consistent with our hypothesis, the mGFP-VSMCs demonstrated higher expression of PDGFR $\beta$ and KLF4 but lower levels of miR-223 compared with those in the non-DM group, suggesting that DM platelets with deficient miR-223 infiltration of VSMCs failed to attenuate the increased expression of PDGFR $\beta$ and KLF4. The present study sheds light on a possible cause for the severe intimal hyperplasia often observed with DM $(29,34)$. Our studies also provide some insight into additional miRNA-based therapeutic targets to prevent vascular disease, particularly in DM patients.

Implications for other vascular injuries. VSMC phenotypic switching also plays a crucial role not only in mechanical injury repair, such as balloon angioplasty (or femoral wire injury), but in the pathogenesis of atherosclerosis. VSMCs residing in normal arteries exhibit a contractile phenotype with low proliferative rate $(35,36)$. However, atherosclerosis and vascular injury lead to VSMC migration and proliferation (37). Around atherosclerotic regions, platelets are activated by the exposed collagen and shear stress $(38,39)$, resulting in the release of PDGF and other bioactive proteins, which induce VSMC dedifferentiation, at least in part leading to intimal hyperplasia. Targeting the mechanisms by which platelet miRNAs regulate the gene expression of VSMCs represents a potential therapeutic strategy for treatment of many vascular smooth muscle-related vascular diseases.

\section{Methods}

Platelet purification and activation. Human platelets were isolated from venous blood of consenting volunteers (healthy and DM subjects) at Yale University School of Medicine (Human Investigation Committee no. 1005006865) (Supplemental Table 1) and prepared as previously described (40). Briefly, platelet-rich plasma (PRP) was obtained by centrifugation at $250 \mathrm{~g}$ for 15 minutes, and platelets were sedimented at $1000 \mathrm{~g}$ for 15 minutes and then resuspended at $10^{8}$ platelets $/ \mathrm{ml}$ in HEPES-Tyrode's buffer. Platelet activation was induced upon incubation with $0.1 \mathrm{U} / \mathrm{ml}$ thrombin (Sigma-Aldrich) for 15 or 60 minutes at $37^{\circ} \mathrm{C}$ with gentle agitation. Platelet activation was inhibited by the addition of $20 \mathrm{mM}$ EDTA, and platelets were pelleted by centrifugation at $3200 \mathrm{~g}$ for 10 minutes. For murine platelets, blood was drawn from the right cardiac ventricle into $1.8 \%$ sodium citrate buffer ( $\mathrm{pH}$ 7.4) and diluted with an equal volume of HEPES/Tyrode's buffer. PRP was prepared by centrifugation at $100 \mathrm{~g}$ for 10 minutes, and then washed platelets were prepared from PRPs by centrifugation at $5000 \mathrm{~g}$ for 2 minutes. Platelet pellets were resuspended in HEPES/Tyrode's buffer (41).

Cell culture. Primary human arterial smooth muscle cells (VSMCs) were obtained from Cell Applications at passage 3. Cells were grown in smooth muscle cell media supplemented with $10 \%$ FBS, smooth muscle cell growth supplement, and 1\% penicillin-streptomycin (Gibco). MEG-01 cells (ATCC) were cultured in Dulbecco's modified RPMI 1640 medium (Gibco) supplemented with $4.5 \%$ (vol/vol) L-glutamine (Gibco) and 10\% (vol/vol) FBS (Invitrogen). Primary mouse VSMCs were isolated from $\mathrm{WT}$ and $\mathrm{mT} / \mathrm{mG}$ mouse aortas by enzymatic digestion and cultured as described previously (42). VSMCs were cocultured with or without RPs or thrombin-activated platelets (APs). To determine the functional role of target miRNAs in VSMCs, VSMCs were transfected with miR-143 mimic, miR-145 mimic, miR-223 mimic, or NC (GenePharma) at $100 \mathrm{nM}$ by using Lipofectamine RNAiMAX reagent (Invitrogen) according to the manufacturer's protocols. To restore the expression of miR-143, miR-145, and miR-223 in PLPs derived from HG-treated MEG-01 cells, MEG-01 cells were simultaneously transfected with miR-143, miR-145, and miR-223 mimics at $100 \mathrm{nM}$ by using Lipofectamine RNAiMAX reagent according to the manufacturer's protocols. After that, PLPs were collected from thrombopoietinstimulated (TPO-stimulated) (Life Technologies) MEG-01 cells and cocultured with VSMCs. The proteins were collected after transfection for 48 hours. The sequences are listed in Supplemental Table 2.

CCK8 assay. Cell proliferation was analyzed using a Cell Counting Kit-8 (CCK8; Dojindo). After treatment as described above, VSMCs were incubated with $10 \mu \mathrm{l}$ WST- 8 in $100 \mu \mathrm{l}$ culture medium for 2 hours at $37^{\circ} \mathrm{C}$. The absorbance of each sample was measured at $450 \mathrm{~nm}$ with a microplate reader (Thermo Fisher Scientific).

BrdU incorporation assay. VSMCs were cultured $\left(10^{4}\right.$ per well) in 96-well plates overnight, followed by serum starvation for 24 hours. Starved VSMCs were treated as described above for 48 hours. Cell proliferation was also evaluated using a BrdU Cell Proliferation Assay Kit (Millipore) according to the manufacturer's instructions. Briefly, cells were incubated with BrdU for 12 hours before measurement. The cells were fixed and DNA denatured using fixing solution. After fixation, cells were labeled with a peroxidase-conjugated BrdU antibody, followed by incubation with the peroxidase substrate. Finally, acid Stop Solution was added into each well to stop the reaction. The absorbance of the samples was measured by a microplate reader at $450 \mathrm{~nm}$ $\left(\mathrm{OD}_{450}\right)$ with reference measurement at $550 \mathrm{~nm}\left(\mathrm{OD}_{550}\right)$. Absorbance $\left(\mathrm{A}_{450 \mathrm{~nm}}-\mathrm{A}_{550 \mathrm{~nm}}\right)$ values representing cell proliferation ability were compared between treatments.

miRNA sequencing and data analysis. Total RNA was extracted using a miRNeasy Mini Kit (QIAGEN) according to the manufacturer's protocol. RNA molecules (18 30 nt) were enriched by PAGE. 3' Adapters were then added, and the RNA molecules (36 44 nt) were enriched. The 5 adapters were then ligated to the RNAs as well. The ligation products were amplified by RT-PCR. The PCR products with 140-160 bp were enriched to generate a cDNA library and sequenced using Illumina HiSeq 2500 by Gene Denovo Biotechnology Co. The raw data were 
A

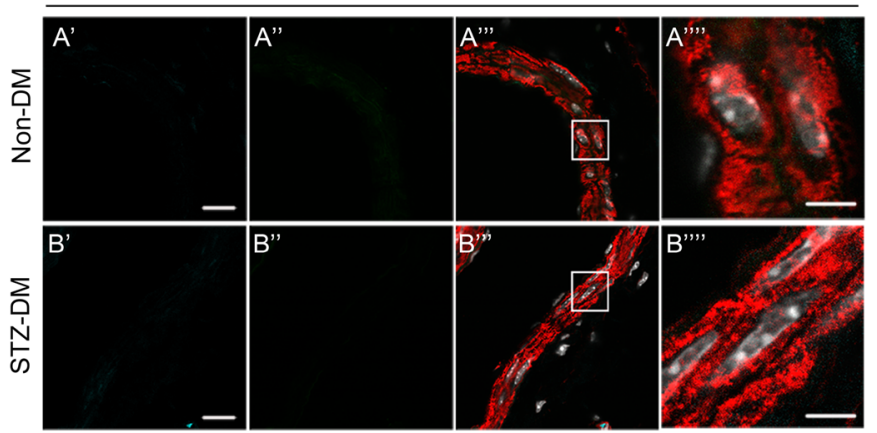

B

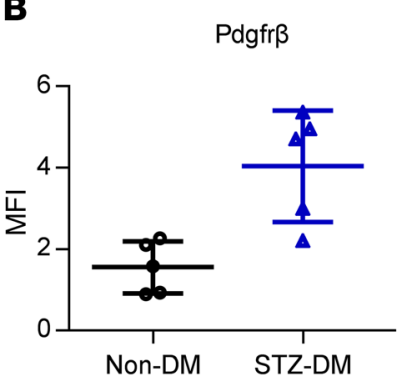

c

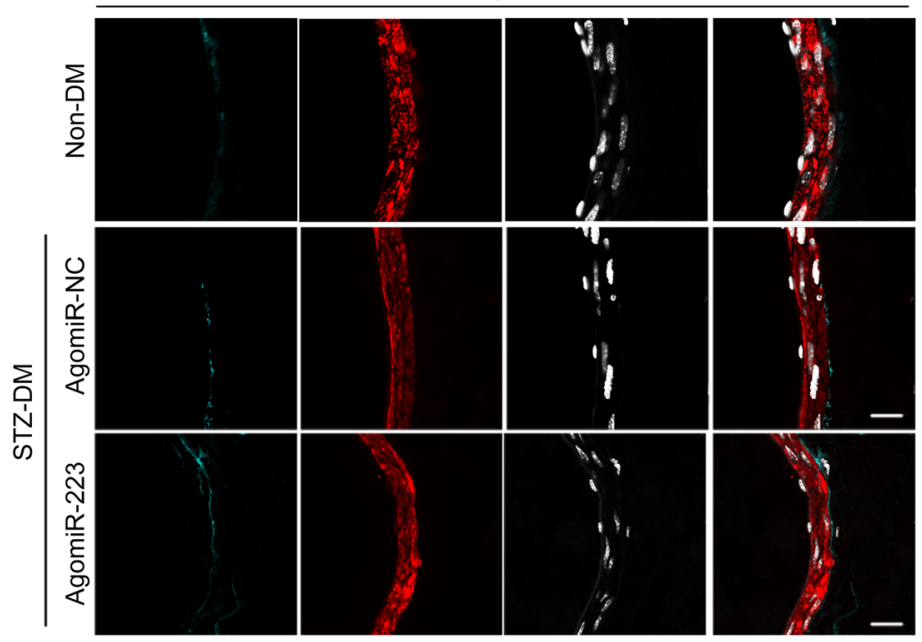

Injured

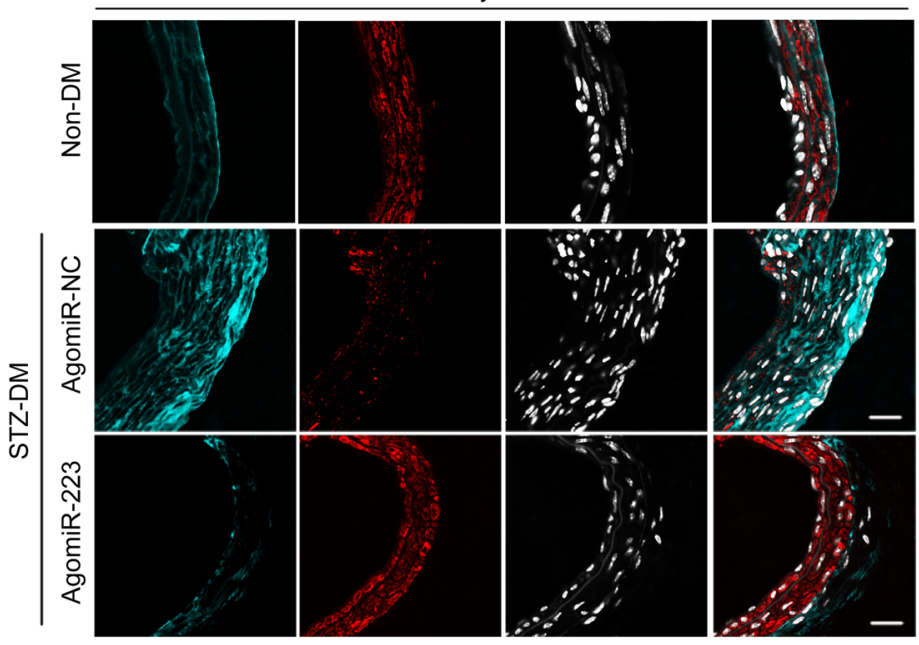

Pdgfrß / Acta2 / DAP
Injured

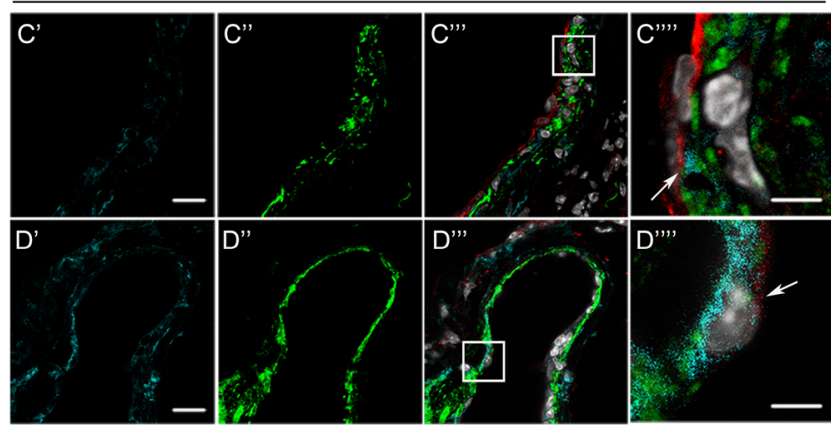

Pdgfr / / mGFP / Acta2 /DAP |

D

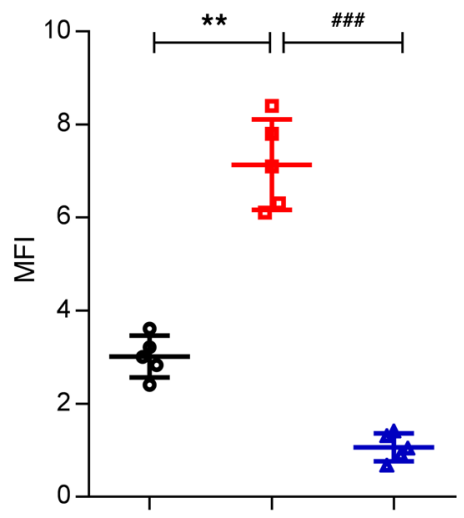

Non-DM AgomiR-NC AgomiR-223 STZ-DM
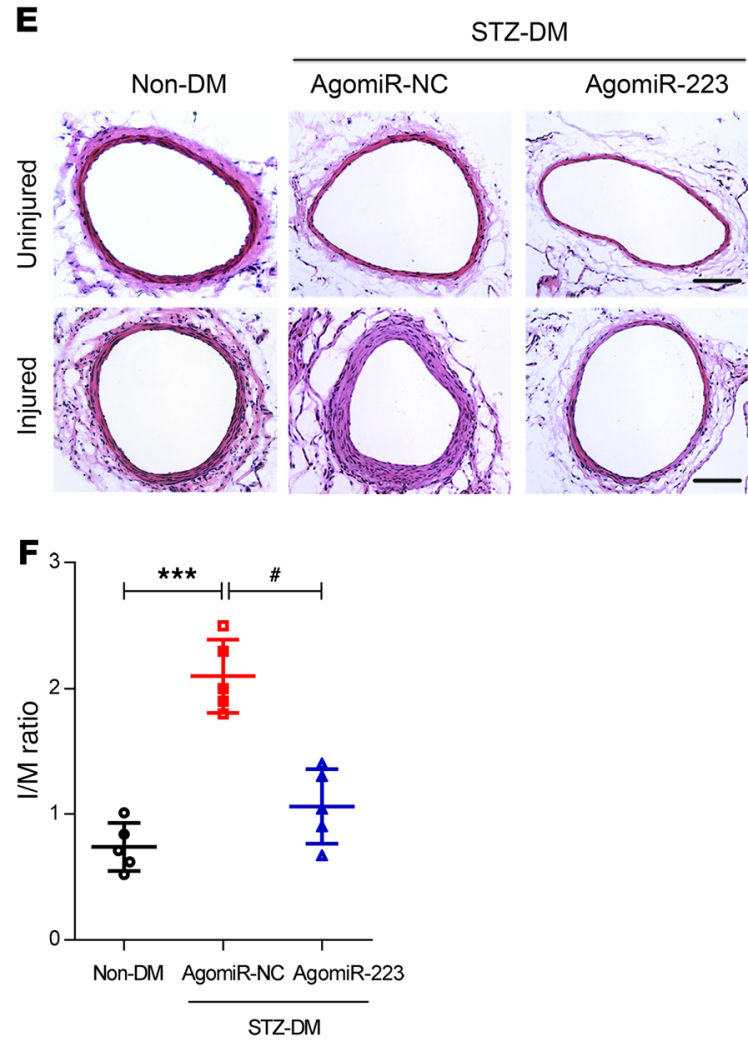
Figure 6. Platelet-derived miR-223 is responsible for inhibition of neointima formation in diabetic mice after femoral artery wire injury.

(A) Representative images of Pdgfr $\beta$ expression in uninjured and injured femoral arteries of PF4-cre:mT/mG mice under non-DM or STZ-DM conditions $(n=5)$. The injured femoral arteries were harvested on the seventh day after injury. Scale bars: $20 \mu \mathrm{m}$. (B) Quantification of Pdgfr $\beta$ expression in MCFP-positive VSMCs in the injured femoral arteries $(n=5)$. Data are presented as mean $\pm \mathrm{SD}$. ${ }^{*} P<0.05$ vs. non-DM. (C) Representative immunofluorescence of Pdgfr $\beta$ in uninjured or injured femoral arteries from non-DM mice and STZ-DM mice treated with AgomiR-NC or AgomiR-223 at 4 weeks after wire injury $(n=5)$. Green, Pdgfr $\beta$; blue, DAPI that reflects total cells; red, Acta2 in VSMCs. Scale bars: $20 \mu \mathrm{m}$. (D) Quantification of Pdgfr $\beta$ expression in VSMCs in the injured femoral arteries $(n=5)$. Data are presented as mean \pm SD. ${ }^{* *} P<0.01$ vs. non-DM; ${ }^{\# \#} P<0.001$ vs. STZ-DM mice treated with AgomiR-NC. (E) H\&E staining of serial cross sections from femoral arteries from non-DM mice and STZ-DM mice treated with AgomiR-NC or AgomiR-223 at 4 weeks after injury $(n=5)$. Scale bars: $100 \mu \mathrm{m}$. (F) Morphometric measurements of I/M ratio in the injured femoral arterial sections $(n=5)$. Data are presented as mean \pm SD of I/M ratio. ${ }^{* * *} P<0.001$ vs. non-DM; ${ }^{\#} P<0.05$ vs. STZ-DM mice treated with AgomiR-NC. Statistical significance was determined using parametric $t$ test (B) and 1-way ANOVA followed by Tukey-Kramer multiplecomparisons test ( $\mathbf{D}$ and $\mathbf{F})$.

processed using Illumina Genome Analyzer Pipeline software and subjected to data filtration. After filtering the low-quality reads and trimming the adaptor sequences, clean reads were obtained. Expression levels of total miRNA - consisting of existing miRNA, known miRNA, and novel miRNA - were calculated and normalized to transcripts per million (TPM). Heatmaps of existing miRNA, known miRNA, and novel miRNA were drawn to display miRNA expression levels in different samples and to cluster miRNAs with similar expression pattern. Those miRNAs with a fold change greater than 1.5 and $P$ value less than or equal to 0.05 were regarded as significant differentially expressed. Results from the sequencing were deposited in the NCBI's Sequence Read Archive database (SRA study SRP135734).

Isolation of RNA and miRNA for qPCR. Total RNA, including miRNA, was isolated from cells using the miRNeasy RNA isolation kit (QIAGEN). RNA and miRNA were reverse transcribed using a PrimeScript RT Reagent Kit (Takara) and Hairpin-it microRNA and U6 snRNA Normalization RT-PCR Quantitation Kit (GenePharma), respectively. RNA was extracted from plasma and serum using a miRNeasy Serum/Plasma Kit (QIAGEN). Caenorhabditis elegans cel-miR-39 (GenePharma) was used as the spike-in control. Transcript levels were analyzed by qPCR using the $2^{-\Delta \Delta C t}$ method. Primer sequences are listed in Supplemental Tables 3 and 4.

Labeling of RNA and miRNA in PLPs. MEG-01 cells were incubated with $0.5 \mathrm{mM}$ EU (Life Technologies) in combination with $100 \mathrm{ng} /$ $\mathrm{ml}$ TPO (Life Technologies) for 48 hours (15) to produce PLPs that incorporated EU into their RNA. Isolated PLPs were washed twice in RPMI 1640 medium (Gibco). EU was visualized using a Click-iT RNA Alexa Fluor 488 Imaging Kit (Life Technologies). For labeling miRNA, platelets were transfected with synthetic cel-miR-39 or fluorescently labeled scrambled miRNA (miR-Src-FITC) using Lipofectamine RNAiMAX reagent, and then washed twice to remove the transfection reagent. After transfection, the platelets were activated by $0.1 \mathrm{U} / \mathrm{ml}$ thrombin and then cocultured with VSMCs. The RNAs were harvested from VSMCs after 4 hours of coculture, and the level of miR-39 transfer was determined by qPCR.
Western blot analysis. Protein was extracted from cells using protein lysis buffer and separated using 12\% SDS-PAGE gels. Primary antibodies were as follows: PCNA (Abcam, catalog ab29), KLF4 (GeneTex, GTX 101508), KLF5 (Abcam, ab137676), osteopontin (OPN; Abcam, ab91655), $\alpha$-smooth muscle actin (ACTA2; Abcam, ab119952), transgelin (TAGLN; Abcam, ab14106), smooth muscle calponin (CNN1; Abcam, ab46794), and PDGFR $\beta$ (Abcam, ab32570). GAPDH (Abcam, ab125247) and $\alpha$-tubulin (Sigma-Aldrich, T8203, clone AA13) were used as the loading control for normalization. Densitometry of the bands was quantified using Image Lab software (Bio-Rad). All antibodies were used at a dilution of 1:1000 unless otherwise specified. Full, uncut gels for all the Western blots are shown in the supplemental material.

Immunofluorescence. Cells and tissues were fixed and stained according to standard protocols (42). Primary antibodies included anti-rabbit PDGFR $\beta$ antibody (1:200, Santa Cruz Biotechnology Inc., catalog sc-374573), anti-goat KLF4 antibody (1:200, R\&D Systems, AF3158), and Alexa Fluor 594-conjugated anti-ACTA2 (1:200, Abcam, ab202510). DAPI staining was performed to visualize nuclei. Images were taken on a Leica SP8 (Leica Instruments) confocal microscope with $100 \times$ oil immersion lens. MFI was calculated by Image $(\mathrm{NIH})$ software and subjected to statistical analysis to determine significance.

Luciferase reporter assay. psi-CHECK2-KLF4, -KLF5, or -PDGFR $\beta$ 3'-UTR plasmid was obtained from Addgene (plasmid 31882). HEK293 cells were plated in a 24-well plate and transfected with $1 \mu \mathrm{g}$ plasmid and $50 \mathrm{nM}$ miRNA mimics (miR-143, miR-145, or miR-223 mimic) by using Lipofectamine RNAiMAX reagent. A dual luciferase assay was performed after 48 hours to measure the firefly luciferase conjugated to the $3^{\prime}$-UTR normalized to Renilla luciferase activity according to the manufacturer's instructions (Promega).

Development of the PF4-cre: $m T / m G$ mouse model. Eight- to 12 -week-old mice were used for all studies. Procedures were approved by the IACUC of Yale University. PF4-cre mice [C57BL/6-Tg(PF4icre)Q3Rsko/J, stock 008535] and $\mathrm{mT} / \mathrm{mG}$ mice [B6.129(Cg)-Gt

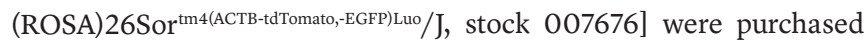
from the Jackson Laboratory and crossed to generate PF4-cre:mT/mG mice. Genotyping was performed via PCR using standard methods. We used PF4-icre $\times$ Gt (ROSA) $26^{\mathrm{mT} / \mathrm{mG}}$ (herein, PF4-mT/mG) reporter mice, in which PF4-icre drives membrane GFP expression in megakaryocytes and platelets, while all other cells are labeled with $\mathrm{mT}$ (43). To induce DM, 8-week-old mice were injected with STZ (50 mg/ $\mathrm{kg}$ ) intraperitoneally for 5 days.

Detection of cre recombination of the ROSA26mT/mG allele. Cre recombination of the ROSA26mT/mG allele was detected according to Hann et al. (44). Briefly, total DNA was extracted from individual tissues using a DNeasy Blood and Tissue extraction kit (QIAGEN). One primer pair (mT-F GCAACGTGCTGGTTATTGTG and mT-R TGATGACCTCCTCTCCCTTG) was designed to generate a 200-bp amplimer from the non-recombined ROSA26mT/mG allele; one primer pair (mT-F GCAACGTGCTGGTTATTGTG and mG-R TGCTCACGGATCCTACCTTC) was designed to generate a 293-bp amplimer from the recombining ROSA26mT/mG allele; and another primer pair (mG-F GTTCGGCTTCTGGCGTGT and mG-R TGCTCACGGATCCTACCTTC) was designed to generate a 376-bp amplimer from the cre-recombined ROSA26mT/mG allele. The sensitivity of the latter primer pair for detecting cre recombination of the ROSA26 $\mathrm{mT} / \mathrm{mG}$ allele was determined by PCR using template genomic DNA from nonrecom- 


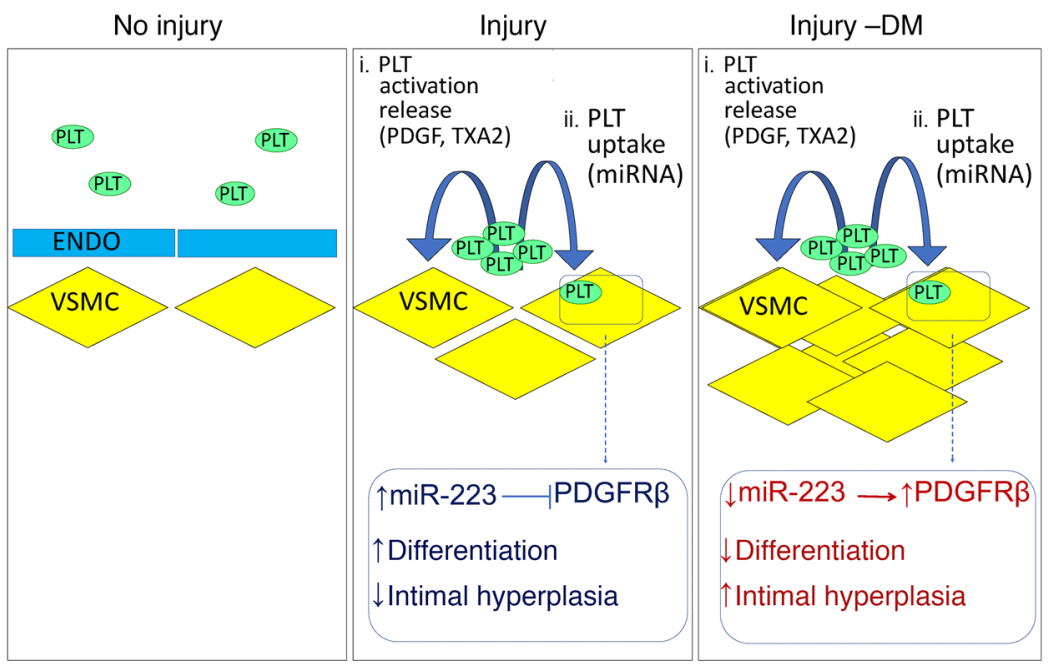

Figure 7. Proposed model for miRNAs transfer in normal and DM platelets into VSMCs in injured vessel. With no vessel injury the endothelial barrier (ENDO) is intact and platelets are quiescent. With injury (as with angioplasty), the endothelial barrier is compromised. Platelets (PLT) are activated and (i) release agents that promote VSMC dedifferentiation; and (ii) are taken up by VSMCs and release miR-223, which applies a brake on VSMCs, preventing excess VSMC repair, reducing intimal hyperplasia. DM platelets have reduced miR223 , resulting in excess VSMC proliferation upon injury. Insets indicate biochemical target for miR-223 including PDGFR $\beta$. The levels of miR-223 are significantly reduced in DM platelets, resulting in upregulation of PDCFR $\beta$, promoting aberrant VSMC dedifferentiation, proliferation, and intimal hyperplasia.

bined mice that contained serially diluted genomic DNA and VSMCs that had completely recombined alleles. The unmixed genomic DNAs were used as negative and positive controls for each primer pair, and the recombination assay was performed using $200 \mathrm{ng}$ template DNA.

Femoral artery wire injury model. Endoluminal injury to the common femoral artery was produced by 3 passages of a $0.15-\mathrm{mm}$ diameter angioplasty guidewire (Advanced Cardiovascular Systems). Nonoperated contralateral femoral arteries were used as controls. Arterial specimens were analyzed in a blindly manner by computerized morphometry (NIH Image 1.60 software). Neointima formation was assessed by H\&E staining, and percent luminal narrowing and $\mathrm{I} / \mathrm{M}$ ratio were calculated as previously described (45). Intraobserver and interobserver variation coefficients for serial measurements of morphometric parameters were $<0.5 \%$. Arteries with occlusive thrombi were not included in the morphometric analysis.

Animal care and use in accordance with institutional guidelines. Global miR-223-KO mice were purchased from the Jackson Laboratory and backcrossed to $\mathrm{C} 57 \mathrm{BL} / 6$ mice for $\geq 5$ generations. In this strain, the allele replaces the entire coding region of the micro RNA-223 (Mir223) gene with a frt-flanked neomycin (neo) resistance cassette, abolishing gene function. These mice also harbor the CD45.1 (Ly5.1 or Ptprca) allele rather than the CD45.2 (Ly5.2 or Ptprcb) allele normally present in C57BL/6 mice. For perivascular delivery of miR-223, wire-injured femoral arteries were randomly assigned to the miR-223 or miR-NC AgomiR group, and pluronic gel was applied as described in previous studies (46). Briefly, after wire injury, $100 \mu \mathrm{l}$ of $30 \%$ pluronic gel containing chemically modified and cholesterol-conjugated $2.5 \mathrm{nmol} \mathrm{miR-223}$ or scrambled (miR-NC) AgomiRs was applied perivascularly to injured femoral arteries. The micrON miRNA agomiRs were purchased from Shanghai GenePharma Co.

FISH. FISH was performed according to the manufacturer's protocol. Briefly, frozen sections were fixed with $4 \%$ paraformaldehyde for 30 minutes and washed with PBS (Gibco). Fixed sections were treated with proteinase $\mathrm{K}$ at $37^{\circ} \mathrm{C}$ for 10 minutes, followed by dehydration in ethanol for 5 minutes. The probe targeting miR-143-3p, miR-145-5p, or miR-223-3p (BioSense) was then added to the respective section. Hybridization was performed in a humid chamber at $42^{\circ} \mathrm{C}$ overnight. Post-hybridization wash was first performed with $50 \%$ formamide with $2 \times$ saline sodium citrate at $42^{\circ} \mathrm{C}$, and then washed with $2 \times$ saline sodium citrate at room temperature to remove nonspecific and repetitive RNA hybridization. Slides were counterstained with DAPI (Vector Laboratories) and examined with a Leica SP8 confocal microscope. MFI was calculated by ImageJ software and subjected to statistical analysis for determination of significance.

Electron microscopy. For ultrastructural analysis, VSMCs were cocultured with APs for 4, 24, and 48 hours, and then fixed with $2.5 \%$ glutaraldehyde for more than 2 hours. The fixed cells were then rinsed with $0.1 \mathrm{M}$ sodium cacodylate buffer 6 times (30 minutes each) and postfixed with $1 \%$ osmium tetroxide for 1 hour at $4^{\circ} \mathrm{C}$. Cells were washed 3 times in $0.1 \mathrm{M}$ sodium cacodylate buffer and dehydrated with a graded series of ethanol $(30 \%, 50 \%$, $70 \%, 90 \%$, and $100 \%$; 5 minutes each), followed by embedding in Epon812 and ultrathin sectioning. After uranyl acetate and lead citrate staining, ultrathin sections were examined with the transmission electron microscope FEI CM100 (Japan Electron Optics Laboratory). Images were captured with an Advantage CCD camera using iTEM software (Olympus).

Statistics. For normally distributed data, values are expressed as mean $\pm \mathrm{SD}$. Parametric $t$ test was performed for comparing 2 groups. One-way ANOVA followed by Tukey-Kramer multiplecomparisons test was performed for comparing 3 or more groups within the same study. For data that were not distributed normally, values were expressed as medians and IQRs. Mann-Whitney $U$ test was used to analyze differences between indicated groups. All statistical analysis was performed using Prism 7 software (GraphPad). A difference at $P<0.05$ was considered significant.

Study approval. The animals were housed at the Yale Animal Facility under the supervision of Yale Animal Resources Center (YARC) and the animal faculty manager. All procedures were approved by the Yale IACUC under animal protocol 11413 and the IACUC of Guangzhou Medical University (no. SYXK2016-0168). All human blood studies were approved by the Yale Human Investigation Committee (protocol 1005006865). Informed consent was obtained from each subject, and the human studies conformed to the principles set out in the World Medical Association (WMA) Declaration of Helsinki and the US National Commission for the Protection of Human Subjects of Biomedical and Behavioral Research Belmont Report. 


\section{Author contributions}

ZZ, JH, and WHT conceptualized the study; ZZ, LX, XF, ACO, TY, MS, YZ, XP, YX, LP, XG, SKC, and WHT developed methodology; ZZ, LX, XF, MS, RL, JH, and WHT performed investigations; ZZ, LX, and WHT wrote the original manuscript draft; KAM, RL, JH, and WHT reviewed and edited the manuscript; RL, JH, and WHT acquired funding; JH and WHT provided resources and supervised the study.

\section{Acknowledgments}

WHT is supported by the Guangdong Outstanding Young Scientist Fund (grant 2016A030306049). This work was supported by the National Natural Science Foundation of China (grant 81670117), the Guangdong Province Science and Technology Project (grant
2017A050506008), and by the Guangzhou Science and Technology Project (grant 201707010270). JH and this work were also supported in part by grants from the NHLBI (HL122815, HL117798, and HL115247). RL is supported by a Future Leader Fellowship (award ID 101856) from the National Heart Foundation of Australia.

Address correspondence to: John Hwa, Yale Cardiovascular Research Center, Section of Cardiovascular Medicine, Department of Internal Medicine, Yale School of Medicine, 300 George St., New Haven 06511, Connecticut, USA. Phone: 203.737.5583; Email: john.hwa@yale.edu. Or to: Wai Ho Tang, Institute of Pediatrics, Guangzhou Women and Children's Medical Center, Guangzhou Medical University, Guangzhou, Guangdong, 510623, China. Phone: 86.020.38076327; Email: waiho.tang@gwcmc.org.
1. Zollikofer CL, et al. Transluminal angioplasty evaluated by electron microscopy. Radiology. 1984;153(2):369-374.

2. Liu MW, Roubin GS, King SB 3rd. Restenosis after coronary angioplasty. Potential biologic determinants and role of intimal hyperplasia. Circulation. 1989;79(6):1374-1387.

3. Broos K, Feys HB, De Meyer SF, Vanhoorelbeke $\mathrm{K}$, Deckmyn H. Platelets at work in primary hemostasis. Blood Rev. 2011;25(4):155-167.

4. Leslie M. Cell biology. Beyond clotting: the powers of platelets. Science. 2010;328(5978):562-564.

5 . Shi N, Chen SY. Smooth muscle cell differentiation: model systems, regulatory mechanisms, and vascular diseases. J Cell Physiol. 2016;231(4):777-787.

6. Pavic G, et al. Thrombin receptor proteaseactivated receptor 4 is a key regulator of exaggerated intimal thickening in diabetes mellitus. Circulation. 2014;130(19):1700-1711.

7. Sobel BE. Acceleration of restenosis by diabetes: pathogenetic implications. Circulation. 2001;103(9):1185-1187.

8. Kitoga M, et al. Coronary in-stent restenosis in diabetic patients after implantation of sirolimus or paclitaxel drug-eluting coronary stents. Diabetes Metab. 2008;34(1):62-67.

9. Lindsay CR, Edelstein LC. MicroRNAs in platelet physiology and function. Semin Thromb Hemost. 2016;42(3):215-222.

10. Diehl P, et al. Microparticles: major transport vehicles for distinct microRNAs in circulation. Cardiovasc Res. 2012;93(4):633-644.

11. Laffont B, et al. Activated platelets can deliver mRNA regulatory Ago $2 \cdot$ microRNA complexes to endothelial cells via microparticles. Blood. 2013;122(2):253-261.

12. Laffont B, et al. Platelet microparticles reprogram macrophage gene expression and function. Thromb Haemost. 2016;115(2):311-323.

13. Gidlöf $\mathrm{O}$, et al. Platelets activated during myocardial infarction release functional miRNA, which can be taken up by endothelial cells and regulate ICAM1 expression. Blood. 2013;121(19):3908-3917, S1.

14. Risitano A, Beaulieu LM, Vitseva O, Freedman JE. Platelets and platelet-like particles mediate intercellular RNA transfer. Blood. 2012;119(26):6288-6295.
15. Kirschbaum M, Karimian G, Adelmeijer J, Giepmans BN, Porte RJ, Lisman T. Horizontal RNA transfer mediates platelet-induced hepatocyte proliferation. Blood. 2015;126(6):798-806.

16. Michael JV, et al. Platelet microparticles infiltrating solid tumors transfer miRNAs that suppress tumor growth. Blood. 2017;130(5):567-580.

17. Plé H, Landry P, Benham A, Coarfa C, Gunaratne $\mathrm{PH}$, Provost $\mathrm{P}$. The repertoire and features of human platelet microRNAs. PLOS ONE. 2012;7(12):e50746.

18. Landry P, Plante I, Ouellet DL, Perron MP, Rousseau G, Provost P. Existence of a microRNA pathway in anucleate platelets. Nat Struct Mol Biol. 2009;16(9):961-966.

19. Nagalla S, et al. Platelet microRNA-mRNA coexpression profiles correlate with platelet reactivity. Blood. 2011;117(19):5189-5197.

20. Cheng Y, et al. MicroRNA-145, a novel smooth muscle cell phenotypic marker and modulator, controls vascular neointimal lesion formation. Circ Res. 2009;105(2):158-166.

21. Xin M, et al. MicroRNAs miR-143 and miR-145 modulate cytoskeletal dynamics and responsiveness of smooth muscle cells to injury. Genes Dev. 2009;23(18):2166-2178.

22. Cordes KR, et al. miR-145 and miR-143 regulate smooth muscle cell fate and plasticity. Nature. 2009;460(7256):705-710.

23. Johnnidis JB, et al. Regulation of progenitor cell proliferation and granulocyte function by microRNA-223. Nature. 2008;451(7182):1125-1129.

24. Fejes Z, et al. Hyperglycaemia suppresses microRNA expression in platelets to increase P2RY12 and SELP levels in type 2 diabetes mellitus. Thromb Haemost. 2017;117(3):529-542.

25. Elgheznawy A, et al. Dicer cleavage by calpain determines platelet microRNA levels and function in diabetes. Circ Res. 2015;117(2):157-165.

26. Millette E, Rauch BH, Defawe O, Kenagy RD, Daum G, Clowes AW. Platelet-derived growth factor-BB-induced human smooth muscle cell proliferation depends on basic FGF release and FGFR-1 activation. Circ Res. 2005;96(2):172-179.

27. Shi G, et al. Platelet factor 4 mediates vascular smooth muscle cell injury responses. Blood. 2013;121(21):4417-4427.

28. Braig D, et al. Transitional changes in the CRP structure lead to the exposure of proinflammatory binding sites. Nat Commun. 2017;8:14188.

29. Yoshida T, Kaestner KH, Owens GK. Conditional deletion of Krüppel-like factor 4 delays downregulation of smooth muscle cell differentiation markers but accelerates neointimal formation following vascular injury. Circ Res. 2008;102(12):1548-1557.

30. Karvinen H, et al. PDGF-C and -D and their receptors PDGFR-alpha and PDGFR-beta in atherosclerotic human arteries. Eur J Clin Invest. 2009;39(4):320-327.

31. Tan M, et al. Thrombin stimulated plateletderived exosomes inhibit platelet-derived growth factor receptor-beta expression in vascular smooth muscle cells. Cell Physiol Biochem. 2016;38(6):2348-2365.

32. Davis-Dusenbery BN, et al. Down-regulation of Kruppel-like factor-4 (KLF4) by microRNA$143 / 145$ is critical for modulation of vascular smooth muscle cell phenotype by transforming growth factor-beta and bone morphogenetic protein 4. J Biol Chem. 2011;286(32):28097-28110.

33. Legrand V. Therapy insight: diabetes and drug-eluting stents. Nat Clin Pract Cardiovasc Med. 2007;4(3):143-150.

34. Qi W, et al. SHP-1 activation inhibits vascular smooth muscle cell proliferation and intimal hyperplasia in a rodent model of insulin resistance and diabetes. Diabetologia. 2017;60(3):585-596.

35. Newby AC, George SJ. Proliferation, migration, matrix turnover, and death of smooth muscle cells in native coronary and vein graft atherosclerosis. Curr Opin Cardiol. 1996;11(6):574-582.

36. Bennett MR, Sinha S, Owens GK. Vascular smooth muscle cells in atherosclerosis. Circ Res. 2016;118(4):692-702.

37. Alexander MR, Owens GK. Epigenetic control of smooth muscle cell differentiation and phenotypic switching in vascular development and disease. Annu Rev Physiol. 2012;74:13-40.

38. Strony J, Beaudoin A, Brands D, Adelman B. Analysis of shear stress and hemodynamic factors in a model of coronary artery stenosis and thrombosis. Am J Physiol. 1993;265(5 Pt 2):H1787-H1796.

39. Mailhac A, et al. Effect of an eccentric severe stenosis on fibrin(ogen) deposition on severely damaged vessel wall in arterial thrombosis. Relative contribution of fibrin(ogen) and platelets. Circulation. 1994;90(2):988-996. 
40. Tang WH, et al. Glucose and collagen regulate human platelet activity through aldose reductase induction of thromboxane. JClin Invest. 2011;121(11):4462-4476.

41. Tang WH, et al. Aldose reductase-mediated phosphorylation of 553 leads to mitochondrial dysfunction and damage in diabetic platelets. Circulation. 2014;129(15):1598-1609.

42. Liu R, et al. ARHGAP18 protects against thoracic aortic aneurysm formation by mitigating the synthetic and proinflammatory smooth muscle cell phenotype. Circ Res. 2017;121(5):512-524.

43. Muzumdar MD, Tasic B, Miyamichi K, Li L, Luo L. A global double-fluorescent Cre reporter mouse. Genesis. 2007;45(9):593-605.

44. Hann S, Kvenvold L, Newby BN, Hong M, Warman ML. A Wisp3 Cre-knockin allele produces efficient recombination in spermato- cytes during early prophase of meiosis I. PLoS ONE. 2013;8(9):e75116.

45. Liu R, et al. Ten-eleven translocation-2 (TET2) is a master regulator of smooth muscle cell plasticity. Circulation. 2013;128(18):2047-2057.

46. Yang F, et al. miR-22 is a novel mediator of vascular smooth muscle cell phenotypic modulation and neointima formation. Circulation. 2018;137(17):1824-1841. 